\title{
Quality water not everywhere: Exploratory Analysis of Water Quality Across Ebocha-Obrikom Oil and Gas Flaring Area in the Core Niger Delta Region of Nigeria
}

Morufu Olalekan Raimi ( $\nabla$ ola07038053786@gmail.com )

Niger Delta University https://orcid.org/0000-0001-5042-6729

Olawale Henry Sawyerr

Kwara State University, Nigeria

Clinton Ifeanyichukwu Ezekwe

University of Port Harcourt

Opasola Afolabi Olaniyi

Kwara State University

\section{Research Article}

Keywords: Decision making, Stakeholders, Indigenous populations, Wasted health, Water quality, Exploratory analysis, Core Niger Delta

Posted Date: October 4th, 2021

DOI: https://doi.org/10.21203/rs.3.rs-953146/v1

License: (9) This work is licensed under a Creative Commons Attribution 4.0 International License.

Read Full License 


\section{Abstract}

\section{Background:}

In Rivers State, Nigeria, Indigenous communities of Ebocha-Obrikom have nearly worst drinking water quality in the province with concerns extending from worsening water quality to deficiency of support from oil companies operating in the environment. The minute water is suspected, or recognized of being unsafe or hazardous to human consumption, community people are likely to be positioned under a wasted health.

\section{Objectives:}

To compare water quality parameters in the vicinity of Gas Flaring Area of Ebocha-Obrikom of Rivers State with that of the recommended standards.

\section{Methods:}

The research utilized standard analytical procedures. All sampling, conservation, transportation and analysis followed standard procedures described in APHA (2012). All the samples collected were transported to the laboratory through keeping in an icebox to prevent degradation of the organic substances.

\section{Results:}

Result depicts that Turbidity, DO, BOD, COD, TSS, Magnesium, Iron, Cadmium, Lead, Chromium, and Nickel exceeded the desirable limit meant for drinking purpose as well as could potentially pose threats toward human society. Hence, remain unsuitable for drinking, as the inhabitants were more vulnerable for their total lifetime period of exposure through continuous consumption of unsuitable drinking water.

\section{Conclusion:}

It is recommended that the local government environmental health officers and other regulatory agencies frequently monitor the levels of these pollutants within the area and also ensure strict adherence to guidelines to ensure a healthy environment. As exposure to the above stated parameters can have a remarkable impact on human health living in the vicinity of the gas flaring area by drinking water around the study area; thus, groundwater needs to treated before using for household purpose or drinking. Thus, this study would help in decision making for stakeholders and relevant authorities in the execution of reasonable groundwater management strategies and remediation plans in the area to protect public and environmental health.

\section{Highlights}


- Groundwater (GW) quality is crucial for indigenous population of Nigeria's oil rich communities, their health, food system, societal stability and welfare.

- This study aims to compare water quality parameters with the recommended standards in the study areas.

- Result depicts that geogenic enrichment of harmful elements like Turbidity, DO, BOD, COD, TSS, Magnesium, Iron, Cadmium, Lead, Chromium, and Nickel etc. exceeded the desirable limit aimed at drinking purpose as well as would likely threaten human society, including safe utilization and rational management of groundwater resources. Thus, remain unsuitable for drinking. These potential toxic elements should be paid attention to when the water is utilized as a domestic water resource.

- The danger of cancer as well as disease risk to millions of individuals living in direct proximity to the gas flaring is a basis for apprehension in its own right, with the impact of those constant gases as well as toxins released to the atmosphere have worldwide consequence.

- Regarding the environmental and social conditions of the area, gas flaring increases the health hazards considerably, firstly through direct release of hazardous chemicals into the atmosphere and secondly through transporting the pollutants into the food chain.

- Aside from the environmental/human effect, the financial cost of groundwater pollution amounts to billions of Naira. Thus, there is need for tougher environmental regulations.

- Government must implement regular water quality monitoring instruments of groundwater quality for the indigenous oil rich society welfare in the core Niger Delta.

\section{Introduction}

In Niger Delta, the availability and drinking water quality remain a reflection of where you reside. Due to its perceived abundance, it has mainly been taken for granted particularly its access and safe drinking water availability (Morufu and Clinton, 2017; Raimi and Sabinus, 2017; Raimi et al., 2017; Olalekan et al., 2018; Premoboere and Raimi, 2018; Olalekan et al., 2019; Raimi et al., 2019; Raimi, 2019; Olalekan et al., 2020). In Niger Delta, the availability as well as quality of drinking water vary according to where you reside (Raimi et al., 2018; Olalekan et al., 2018; Raimi et al., 2019; Gift et al., 2020; Gift and Olalekan, 2020; Olalekan et al., 2020; Okoyen et al., 2020). The water type indigenous populations consume, from treated tap water through to private well water or from bottled water usually consumed by oil workers to untreated sourced water collected and country-specific differences in preferences for water consumption are partly due to socio-demographics as well as perceived health concerns or risks (Olalekan et al., 2018; Afolabi and Raimi, 2021) (Raimi et al., 2017; Raimi and Sabinus, 2017; Olalekan et al., 2018; Raimi, 2019; Olalekan et al., 2019; Olalekan et al., 2020). Besides, advisories remain mostly given as a preventative measure, not necessarily on the basis that water is dangerous or unsafe or the risk is consistent; therefore, the degree of the drinking water risk differs on a case-to-case basis (Olalekan et al., 2020). In spite of these, when drinking water availability or quality is undermined, trustworthy as well as efficient public/environmental health sector risk communication is important to reduce negative health impacts, 
irrespective of community size of the population (Morufu and Clinton, 2017; Raimi and Sabinus, 2017; Raimi et al., 2017; Olalekan et al., 2018; Premoboere and Raimi, 2018; Olalekan et al., 2019; Raimi et al., 2019; Raimi, 2019; Olalekan et al., 2020; Raimi et al., 2020; Adedoyin et al., 2020). Many of them comprise small (under 5000) or extremely small populations (less than 500), have difficulty providing enough and safe drinking water since they exist in a regulatory vacuum due of the policies as well as practices established more than 100 years ago and which continue to apply to this day (Raimi et al., 2019; Olalekan et al., 2019; Suleiman et al., 2019). Likewise, owing to its direct as well as intimate relationship to public as well as environmental health and a vital component in community sustainable development, the quality of drinking groundwater resources has attracted significant attention (Raimi et al., 2017; Morufu and Clinton, 2017; Raimi and Sabinus, 2017; Olalekan et al., 2018; Olalekan et al., 2018; Raimi, 2019; Raimi et al., 2019; Olalekan et al., 2019; Raimi et al., 2019; Gift and Olalekan, 2020; Gift et al., 2020; Olalekan et al., 2020; Afolabi and Raimi, 2021). Groundwater in most urban as well as rural areas of the global south, notably the core Niger Delta region of Nigeria, is regarded one of the largest important sources of potable water (Morufu and Clinton, 2017; Raimi and Sabinus, 2017; Olalekan et al., 2018; Olalekan et al., 2018). Under natural geological circumstances and human actions the quality of the groundwater supplies can be altered (Morufu and Clinton, 2017; Raimi and Sabinus, 2017; Olalekan et al., 2018; Olalekan et al., 2018). Recently, the quality of groundwater has been significantly degraded through mineral activities, industrial as well as agriculture with pollutants infiltrating water resources (Morufu and Clinton, 2017; Raimi and Sabinus, 2017; Olalekan et al., 2018; Olalekan et al., 2018; Afolabi and Raimi, 2021). Thus, the use of contaminated water can thereby raise the danger to human health (Morufu and Clinton, 2017; Raimi and Sabinus, 2017; Olalekan et al., 2018; Olalekan et al., 2018). For several decades it has been recognized that trace levels of specific elements impact plants, animals as well as human life positively or negatively (Olalekan et al., 2021; Raimi et al., 2021). For proper physiological activities of mammals of extremely low concentrations, heavy metals such as manganese, iron, copper, zinc, cobalt, chromium and selenium are necessary (Table 1). These elements are called 'essential trace elements' or 'micronutrients' and their insufficiencies can also lead to plant and animal illness as well as even death. Yet, excessive levels of one or more of these lead to changing physiological functioning in the cells of the body. There is no such recognized basic role as necessary trace elements as other elements including: Ba, $\mathrm{Cd}, \mathrm{Ti}, \mathrm{Hg}, \mathrm{Pb}, \mathrm{Ag}, \mathrm{Sb}$ as well as As causes toxicity exceeding a certain degree of tolerance. In terms of potential hazard as well as occurrence in contaminated soils, $\mathrm{Zn}, \mathrm{Hg}, \mathrm{Cd}, \mathrm{Cr}, \mathrm{Pb}$ as well as As are the major heavy metal (Raimi et al., 2021). 
Table 1

Nutritive Attributes of Trace Metals

\begin{tabular}{|c|c|c|c|c|}
\hline$S / N$ & Trace Metals & $\begin{array}{l}\text { Deficiency } \\
\text { Causing } \\
\text { Factors }\end{array}$ & $\begin{array}{l}\text { Affected Body } \\
\text { Functions }\end{array}$ & $\begin{array}{l}\text { Deficiency } \\
\text { Symptoms }\end{array}$ \\
\hline 1. & Zinc & $\begin{array}{l}\text { Excessive } \\
\text { intake of } \\
\text { Copper and } \\
\text { Calcium, } \\
\text { Alcohol, lack } \\
\text { of phosphorus, } \\
\text { phytic acid, } \\
\text { oral } \\
\text { contraceptives }\end{array}$ & $\begin{array}{l}\text { Burn and Wound } \\
\text { healing, Carbohydrate } \\
\text { digestion Prostate gland } \\
\text { function, Reproductive } \\
\text { organ growth and } \\
\text { maturity, Vitamin B, P } \\
\text { and protein metabolism }\end{array}$ & $\begin{array}{l}\text { Delayed sexual maturity, } \\
\text { Fatique, loss of taste and } \\
\text { appetite, prolonged wound } \\
\text { healing, Retarred growth, } \\
\text { Sterility, Atherosclerosis } \\
\text { Diabetes, Improper } \\
\text { metabolism, Baldness, } \\
\text { Infertility }\end{array}$ \\
\hline 2. & Copper & $\begin{array}{l}\text { Excessive Zn, } \\
\text { Mn, Cd Intake, } \\
\text { Oral } \\
\text { Concentration }\end{array}$ & $\begin{array}{l}\text { Bone Formation Colour } \\
\text { of hair and Skin, } \\
\text { Haemoglobin and RBC } \\
\text { formation, Emotional } \\
\text { states }\end{array}$ & $\begin{array}{l}\text { General weakness, Impaired } \\
\text { respiration, Skin sores, } \\
\text { Diarrhoea, Baldness, Copper } \\
\text { deficiency, Anaemia. }\end{array}$ \\
\hline 3. & Chromium & $\begin{array}{l}\text { Excessive Iron } \\
\text { intake }\end{array}$ & $\begin{array}{l}\text { Glucose metabolism, } \\
\text { Blood sugar lever, } \\
\text { Circulatory system }\end{array}$ & $\begin{array}{l}\text { Atherosclerosis, protein-calory } \\
\text { malnutrition, glucose } \\
\text { intolerance, multiple } \\
\text { pregnancies, disturbed amino } \\
\text { acid metabolism. }\end{array}$ \\
\hline 4. & Iron & $\begin{array}{l}\text { Coffee, } \\
\text { Excessive } P \\
\text { Zn, Cu and } \mathrm{Mn} \\
\text { intake. }\end{array}$ & $\begin{array}{l}\text { Haemoglobin } \\
\text { production, childhood } \\
\text { growth, stress, disease's } \\
\text { resistance }\end{array}$ & $\begin{array}{l}\text { Breathing problems, Brittle } \\
\text { nails, Pale skin fatique, } \\
\text { constipation, inflamed tongue }\end{array}$ \\
\hline 5. & Magnesium & $\begin{array}{l}\text { Protein, High } \\
\text { intake of } \\
\text { Vitamin D \& } \\
\text { Ca, too much } \\
\text { fat in diet, oral } \\
\text { contraceptives }\end{array}$ & $\begin{array}{l}\text { Acid/alkali balance, } \\
\text { Blood sugar } \\
\text { metabolism Metabolism } \\
\text { of Ca \& Vitamin C } \\
\text { Protein structuring }\end{array}$ & $\begin{array}{l}\text { Sensitivity to noise, } \\
\text { Disorientation, Tooth decay, } \\
\text { Nervousness, Rapid pulse, } \\
\text { Overweight, Tremors, } \\
\text { depression, Heart problems, } \\
\text { Prostate troubles, Stomach, } \\
\text { troubles, Protein-calorie } \\
\text { malnutrition. }\end{array}$ \\
\hline 6. & Nickel & $\begin{array}{l}\text { Vitamin C, Ca, } \\
\text { Sulphur } \\
\text { containing } \\
\text { amino acids }\end{array}$ & $\begin{array}{l}\text { Membrane structure, } \\
\text { Nucleic acid } \\
\text { metabolism, Liver } \\
\text { Reproduction }\end{array}$ & $\begin{array}{l}\text { Brain blood vessel damage, } \\
\text { Liver cirrhosis, Kidney } \\
\text { malfunction, Lung cancer, } \\
\text { Asthma, Nasal passage } \\
\text { cancer. }\end{array}$ \\
\hline 7. & Selenium & $\begin{array}{l}\text { Mercury, Silver, } \\
\text { Arsenic, } \\
\text { Cadmium, } \\
\text { Sulfates. }\end{array}$ & $\begin{array}{l}\text { Pancreatic function } \\
\text { (possible increased } \\
\text { resistance to cancer, } \\
\text { Membrane integrity. }\end{array}$ & $\begin{array}{l}\text { Mercury toxicity, Pancreatic } \\
\text { insufficiency, Cardiac toxicity, } \\
\text { Aging pigments, Haemolytic } \\
\text { problems, Petroxidation of Fat, } \\
\text { Cancer, Muscle wasting. }\end{array}$ \\
\hline
\end{tabular}

Source: Adapted and modified from Olalekan et al., 2018; Olalekan et al., 2020 


\begin{tabular}{|lllll|}
\hline S/N & Trace Metals & $\begin{array}{l}\text { Deficiency } \\
\text { Causing } \\
\text { Factors }\end{array}$ & $\begin{array}{l}\text { Affected Body } \\
\text { Functions }\end{array}$ & $\begin{array}{l}\text { Deficiency } \\
\text { Symptoms }\end{array}$ \\
\hline 8. & Manganese & $\begin{array}{l}\text { High Ca, Iron \& } \\
\text { Phosphorus } \\
\text { intake. }\end{array}$ & $\begin{array}{l}\text { Reproduction, Enzyme } \\
\text { activation, Growth } \\
\text { Production of sex } \\
\text { hormones, Fat, } \\
\text { Carbohydrate, Vitamin B } \\
\text { \& E metabolism, Tissue } \\
\text { respiration }\end{array}$ & $\begin{array}{l}\text { Fatigue, Ear noise, Muscle } \\
\text { coordination failure, Dizziness, } \\
\text { Abnormal carbohydrate, Loss } \\
\text { of hearing, Diabetes. }\end{array}$ \\
\hline 9. Molybdenum & $\begin{array}{l}\text { Copper } \\
\text { sulfates, Zinc, } \\
\text { Methionine } \\
\text { Lead, Vitamin } \\
\text { B }_{12}\end{array}$ & $\begin{array}{l}\text { Liver function, Kidney } \\
\text { function, Blood, Copper } \\
\text { and iron metabolism }\end{array}$ & $\begin{array}{l}\text { Copper deficiency anaemia, } \\
\text { Gout }\end{array}$ \\
\hline Source: Adapted and modified from Olalekan et al., 2018; Olalekan et al., 2020 \\
\hline
\end{tabular}

Generally, rural and small communities (Indigenous population or not) across the Niger Delta region have their own particular sets of drinking water issues, for example: whether local access to water quality monitoring expertise is available (Raimi et al., 2017; Raimi et al., 2017; Morufu and Clinton, 2017; Premoboere and Raimi, 2018; Olalekan et al., 2018); larger private wells percentage in these oil rich communities in the core Niger Delta depend upon these kind of water (Raimi et al., 2017; Raimi et al., 2017; Morufu and Clinton, 2017; Premoboere and Raimi, 2018; Olalekan et al., 2018); improved changing climate susceptibility (Raimi et al., 2018; Morufu et al., 2021); as well as available resources toward responding to emergencies (Raimi et al., 2020; Raimi and Raimi, 2020; Morufu et al., 2021; Raimi et al., 2021; Morufu et al., 2021; Raimi et al., 2021). More particular, researchers discovered that the indigenous population in oil rich communities, has insufficient record keeping, which included factors like the past improvement, the various infrastructure age within the system, as well as [when or why] past water advisories [have occurred], which led to knowledge gaps in the supplies of water as well as distribution systems. The ability toward accessing safe as well as clean drinking water along shorelines of the Niger Delta is further compounded via the geographical and climatic properties of these regions (Raimi et al., 2018; Morufu et al., 2021); for instance, rising temperatures, extreme weather events, coastal erosion, as well as flooding can lead to intrusion (salinization) of salt water, which may damage infrastructure as well as compromise water quality (Odubo and Raimi, 2019; Suleiman et al., 2019; Raimi et al., 2019; Okoyen et al., 2020; Raimi et al., 2021). In summary, the shifting climate and accelerating changes to water supplies will eventually affect usage of water for production, consumption as well as development (Morufu and Clinton, 2017; Raimi and Sabinus, 2017; Raimi et al., 2017; Olalekan et al., 2018; Premoboere and Raimi, 2018; Olalekan et al., 2019; Raimi et al., 2019; Raimi, 2019; Ajayi et al., 2020; Olalekan et al., 2020; Raimi et al., 2021), and while the perception and adaptation of humans to the risk posed by climate change is uncertain, new research indicates complicated connections between environmental stress and human health in relation to changing climate (Raimi et al., 2018; Morufu et al., 2021; Raimi et al., 2021). At the same time, researchers indicate that shoreline communities remain predominantly sensitive to 
water supply modification since there are over a billion individuals residing in the coastal regions globally as well as the population influx toward these places is constantly influenced. This will certainly have a substantial effect on coastal aquifers especially when pumping water from wells for personal usage, drinking, irrigation as well as commercial use (Odubo and Raimi, 2019; Okoyen et al., 2020; Olalekan et al., 2020). Due to Africa low-tech factories which is quickly build, they cause pollution of groundwaters, rivers and even streams. Ignored for economic reasons, this leads to excessive pollution of easily accessible quality water in the country. In particular, factories producing chemical products can release wastewater to the nearest water. This practice, which saves them from the financial burden, imposes an incredible burden on public finance and threatens their habitats (Odipe et al., 2018; Sawyerr et al., 2018; Henry et al., 2019; Omotoso et al., 2021; Raimi et al., 2021). The worsening worldwide water problem is threatening emerging nations safety, stability as well as environmental sustainability. Every year millions die of waterborne illnesses (Raimi et al., 2017; Olalekan et al., 2018; Raimi et al., 2019; Olalekan et al., 2020; Gift and Olalekan, 2020; Gift et al., 2020; Afolabi and Raimi, 2021), however water pollution as well as growing destruction of ecosystem, mostly in the global south (Olalekan et al., 2018; Premoboere and Raimi, 2018; Raimi, 2019; Olalekan et al., 2020; Raimi et al., 2020; Olalekan et al., 2020; Adedoyin et al., 2020; Olalekan et al., 2020; Ajayi et al., 2020; Raimi et al., 2021). In recent decades, there have been growing acceptance of the need to conduct water resource management with an integrated approach, that resource assessment is of fundamental relevance as a foundation for policy making and that necessary assessments must be fully supported by national capacities (Raimi et al., 2019; Omidiji and Raimi, 2019; Olalekan et al., 2020; Adedoyin et al., 2020; Olalekan et al., 2020; Raimi et al., 2020). Decisions from management on poverty reduction, economic growth, food safety as well as the human health of populations and the preservation of critical ecosystems must remain based on optimal possible knowledge of all pertinent systems (Sawyerr et al., 2018; Oluwaseun et al., 2019; Isah et al., 2020; Olalekan et al., 2020; Isah et al., 2020; Morufu, 2021; Hussain et al., 2021; Morufu et al., 2021; Hussain et al., 2021; Raimi et al., 2021). As the overall demand for water grows, the quantity produced, and its overall pollution load are continuously increasing worldwide (Olalekan et al., 2019; Raimi et al., 2019; Olalekan et al., 2020; Afolabi and Raimi, 2021). For human existence, health as well as dignity, water is essential. It is an essential and irreplaceable development resource (Olalekan et al., 2020; Afolabi and Raimi, 2021). In reality, the good of all societies is closely related on sustainable water resources exploitation (Olalekan et al., 2019; Raimi et al., 2019; Olalekan et al., 2020; Afolabi and Raimi, 2021). The demand for this essential resource has been increasing globally, owing among other things, to fast economic development as well as increased demographic growth (Zhang et al., 2019; Raimi, 2019; Raimi et al., 2019; Zhou et al., 2019; Raimi et al., 2021). This will also assist ground water managers as well as policy makers assess the health consequences associated with their own actions, thus this will give policy makers a basis of making sound policy on whether they should base on environmentally friendly development to boost groundwater quality or focus on economic growth alone and would permit aid organizations toward targeting their resources more efficiently (Raimi et al., 2019; Omidiji and Raimi, 2019; Olalekan et al., 2020; Adedoyin et al., 2020; Olalekan et al., 2020; Raimi et al., 2020). It is expected that the result of this study will assist to meet the challenges of achieving improved quality of life and health status in the state, strengthen the already existing strategies to provide health care services in River's state and Nigeria at 
large. Consequently, this study aimed to compare water quality parameters in the vicinity of Gas Flaring Area of Ebocha-Obrikom of Rivers State with that of the recommended standards. Thus, this research may provide valuable information on the heavy metals and other physico-chemical analysis of drinking water associated with the contamination of the ground waters by petroleum products.

\section{Material And Methods}

\section{The Study Area}

The area is placed between latitude $5^{0} 20 \mathrm{~N}-5^{0} 27 \mathrm{~N}$ and longitude $6^{0} 40 \mathrm{E}-6^{0} 46 \mathrm{E}$ is situated in EbochaObrikom (Figure 1). It encompasses Obrikom, Obie, Obor, Ebocha as well as Agip New Base towns all located in Ogba/ Egbema/Ndoni Area of Rivers State (Figure 1). The study research area lies to the North by River Nkissa, by the West, River Orashi, by the East, River Sombrero and by the South Omoku town.

\section{Climate}

The climate of the study area is the equatorial type. Heavy rainfall of about $2500 \mathrm{~mm} /$ annum are experienced in the region. During the year, the rainfall takes around eight (8) months (March to October), and even months deemed dry remain often not devoid of occasional precipitation (Morufu and Clinton, 2017; Raimi and Sabinus, 2017; Olalekan et al., 2018). Temperature is usually high and the mean monthly temperature is about $25^{\circ} \mathrm{C}$ (Morufu and Clinton, 2017; Raimi and Sabinus, 2017; Olalekan et al., 2018).

\section{Vegetation}

The vegetation is dominated by fresh water swamps, spotted areas are characterized by stratified high forest: The vegetation comprises a multitude of evergreen trees that yield tropical hard wood, e.g., mahogany. Smaller palm trees are present, ascending plants such as lianas or rattan palms may be hundreds of meters long and also epiphytes and parasites that grow on other plants. A vast variety of orchids, creepers and ferns thrive below the trees (Morufu and Clinton, 2017; Raimi and Sabinus, 2017; Olalekan et al., 2018). The traditional rainforest vegetation has been replaced in most instances by settlements, farms, fallow and secondary forests, construction of civil structures, oil and natural gas exploration/exploitation. The only areas where primary forests may be found are raffia palm dominated riparian forests along the Sombreiro River, across the Orashi River and sacred groves which is relic of traditional African religion (Morufu and Clinton, 2017; Raimi and Sabinus, 2017; Olalekan et al., 2018).

\section{Topography and Drainage}

The area is actually drained by the Sombreiro on the Eastern flanks and Orashi on the Western flank creating an almost uninterrupted inter-basinal area. The region has a topographic structure virtually flat, as well as is covered by superficial soil, which is made up of silty clays combined with silty sand. There is a clear lack of imposing hills that rise above the whole land surface at a height of about 25 metres above sea level. 
The Orashi River is a prominent feature of the natural drainage system. This river, although an independent river system, which accounts for the drainage of the entire zone, links up with the Niger Delta system during the wet season (flood stage). Due to the more prominent relief of this area, drainage is more efficient and much fewer rivers and creeks drain the area. Backswamp depressions exist which entrap floodwaters and so form perennial lakes in the area. The rivers are prone to flooding which increases the level of water in the water table. The rivers found in the study area are also subjected to tidal flow (Morufu and Clinton, 2017; Raimi and Sabinus, 2017; Olalekan et al., 2018).

\section{Soil}

The soil occurring on this area is part of the recent alluvium of the Niger Delta and shows age differences in a better development of Argillic horizon (clay illuviation). They are dominantly sandy loam on top soil changing to sandy clay loam and then clay in the subsoils. The soil reaction is acid (pH 4.3-5.0) (Ogoni, 2010). The high acidity of the soils is due to the high Aluminium (Al) content of the soils (Alagoa, 2005; Morufu and Clinton, 2017; Raimi and Sabinus, 2017; Olalekan et al., 2018). The soil organic matter content is low as well as could be due to continuous cultivation of the land. The organic matter decreases down the soil profile. Available phosphorus $(P)$ is moderate especially in middle horizons. The soil fertility in term of percent base saturation is high. The soils are mainly Alfisols (Luvisols and Natrosol), Ultisols (Regosols) and Oxisols (orthic Ferralsols). Other soils identified are inceptisols (Gleyic cambisols) and entisols (Albic Arenosols). The soils are Ultisols (Eutric-Paleudalfs) (Alagoa and Derefaka, 2002; Morufu and Clinton, 2017; Raimi and Sabinus, 2017; Olalekan et al., 2018).

\section{Land Use}

The Ebocha-Obrikom area has various patterns of land use. The only perceptible activity of economic importance in the environment is the crude petroleum exploration as well as exploitation. The land use pattern of the area is basically characterized by a number of agricultural activities which include farming, as well as fishing activities which are among the major activities in the area. Some of the crops cultivated include cocoyam, okra, bitter-leaf, cassava, yam etc. The oil palm planting harvesting and processing are also important (Alagoa, 2005; Morufu and Clinton, 2017; Raimi and Sabinus, 2017; Olalekan et al., 2018).

\section{Sample Collection}

The sampling strategy employed for the current research investigation were similar to that utilized by Morufu and Clinton (2017); Raimi and Sabinus (2017); Olalekan et al. (2018) in which sampling was targeted in some vulnerable quarters at a densely populated location. These quarters are places predisposed to all kinds of contamination not only because of their geographical situation but also because of the presence of crude petroleum exploration and exploitation. From the sample location (see table 2 below), extracted water samples from groundwater sources utilized mostly for drinking as well as domestic activities. Sample collections were limited to only groundwater from dug wells or shallow pumping wells built for household uses exclusively. The depth of the wells varies between 10 to $28 \mathrm{~m}$, which is a phreatic aquifer. The sampling locations sites were documented using portable GPS devices. 
In the vicinity of the depot, ground water sources were selected randomly but at various distances from each other for the purpose of this investigation. Furthermore, the samples were manually collected from nine (9) strategic locations in the study area for ground water (boreholes and wells) into previously washed clean plastic sampling bottles after about $20 \mathrm{~min}$ of continuous water flow to ensure adequate aquifer quality that can be appropriately represented.

Table 2. Geographical coordinates of the nine (9) sampling sites (samples).

\begin{tabular}{|c|c|c|c|c|}
\hline $\mathrm{S} / \mathrm{N}$ & Locations & $\begin{array}{l}\text { Altitude } \\
\text { (m) }\end{array}$ & Latitude & Longitude \\
\hline $\begin{array}{l}\text { Site } \\
-1\end{array}$ & $\begin{array}{l}\text { (Borehole) (Opposite ljeoma Quarters. 750m Away from } \\
\text { Agip Gas Flaring Center Ebocha) }\end{array}$ & 10 & $\begin{array}{l}\text { Lat N050 } \\
27^{\prime} 068^{\prime \prime}\end{array}$ & $\begin{array}{l}\text { Long } \\
\text { E006 } \\
480^{\prime \prime} 41^{\prime}\end{array}$ \\
\hline $\begin{array}{l}\text { Site } \\
-2\end{array}$ & $\begin{array}{l}\text { (Borehole) (200m Opposite Agip Gas Flaring Centre } \\
\text { Ebocha and } 50 \mathrm{~m} \text { from Agip Waste Pit) }\end{array}$ & - & $\begin{array}{l}\text { Lat N050 } \\
27^{\prime} 28.7^{\prime \prime}\end{array}$ & $\begin{array}{l}\text { Long } \\
\text { E006 } \\
58.1^{\prime \prime} 41^{\prime}\end{array}$ \\
\hline $\begin{array}{l}\text { Site } \\
-3\end{array}$ & $\begin{array}{l}\text { (Well) (The Apple Hotel } 500 \mathrm{~m} \text { from Waste Pit and 150m } \\
\text { Away from Mgbede Field Oil Well } 7 \text { Ebocha) }\end{array}$ & 16 & $\begin{array}{l}\text { Lat N050 } \\
27^{\prime} 37.5^{\prime \prime}\end{array}$ & $\begin{array}{l}\text { Long } \\
\text { E006 } \\
05.3^{\prime \prime} 42^{\prime}\end{array}$ \\
\hline $\begin{array}{l}\text { Site } \\
-4\end{array}$ & (Well) (1000m Away from the Agip Flare Stack Ebocha) & 22 & $\begin{array}{l}\text { Lat N050 } \\
26^{\prime} 51.5^{\prime \prime}\end{array}$ & $\begin{array}{l}\text { Long } \\
\text { E006 } 41^{\prime} \\
38.8^{\prime \prime}\end{array}$ \\
\hline $\begin{array}{l}\text { Site } \\
-5\end{array}$ & $\begin{array}{l}\text { (Borehole) (Abacha Road Obrikom, 800m Away from } \\
\text { Agip Gas Plant) }\end{array}$ & - & $\begin{array}{l}\text { Lat N050 } \\
23^{\prime} 48.6^{\prime \prime}\end{array}$ & $\begin{array}{l}\text { Long } \\
\text { E006 } \\
36.6^{\prime \prime} 40^{\prime}\end{array}$ \\
\hline $\begin{array}{l}\text { Site } \\
-6\end{array}$ & $\begin{array}{l}\text { (Borehole) (Eagle Base Obor. 2,500m Away from Agip } \\
\text { Gas Plant) }\end{array}$ & 28 & $\begin{array}{l}\text { Lat N050 } \\
23^{\prime} 00.9^{\prime \prime}\end{array}$ & $\begin{array}{l}\text { Long } \\
\text { E006 } 41^{\prime} \\
07.4^{\prime \prime}\end{array}$ \\
\hline $\begin{array}{l}\text { Sites } \\
-7\end{array}$ & $\begin{array}{l}\text { (Well) (Obor Road Obie. } 2000 m \text { Away from Agip Gas } \\
\text { Plant) }\end{array}$ & 24 & $\begin{array}{l}\text { Lat N050 } \\
23^{\prime} 22.5^{\prime \prime}\end{array}$ & $\begin{array}{l}\text { Long } \\
\text { E006 } \\
49.1^{\prime \prime} 40^{\prime}\end{array}$ \\
\hline $\begin{array}{l}\text { Sites } \\
-8\end{array}$ & $\begin{array}{l}\text { (Borehole) (Green River Plant Propagation Centre Naoc } \\
\text { 3000m Away from Agip Gas Plant) }\end{array}$ & 17 & $\begin{array}{l}\text { Lat N050 } \\
24^{\prime} 18.9^{\prime \prime}\end{array}$ & $\begin{array}{l}\text { Long } \\
\text { E006 } 40^{\prime} \\
55.0^{\prime \prime}\end{array}$ \\
\hline $\begin{array}{l}\text { Sites } \\
-9\end{array}$ & (Control) $(35,000 \mathrm{~m}$ from Ebocha) & - & $\begin{array}{l}\text { Lat N50 } \\
4^{\prime} \\
58.1412^{\prime \prime}\end{array}$ & $\begin{array}{l}\text { Long E6 }{ }^{0} \\
39^{\prime} \\
30.4806^{\prime \prime}\end{array}$ \\
\hline
\end{tabular}

All of the samples was obtained during the daytime, from $9.00 \mathrm{am}$ to $4.00 \mathrm{pm}$. Due of insecurity, flooding and COVID-19 lockdown. Night samples were not collected and sampling was performed between 
September, 2019 to August 2020.

\section{Sampling, Preservation and Analysis}

The standard methods outlined in APHA (2012); Morufu and Clinton (2017); Raimi and Sabinus (2017); Olalekan et al. (2018) have been followed by water sampling, conservation, transportation as well as analysis.

\section{Ground Water Collection}

For the analyses of physico-chemical parameters, ground water samples were collected using pre-rinsed 1 litre plastic containers. Pre-rinsed ground water samples for heavy metal analyses were collected with nitric acid of 1 litre containers as well as treated with $2 \mathrm{ml}$ nitric acid (assaying $100 \%$, Trace Metal Grade, Fisher Scientific) prior to storage. These were done to stabilize the metals oxidation conditions. Groundwater samples were collected in two groups of $250 \mathrm{ml}$ glass-stoppered-reagent bottles per sampling location for Biological Oxygen Demand (BOD), and Dissolved Oxygen (DO) determinations. The BOD samples have been properly filled without air trapping as well as the bottles covered in black polythene bags. This was done to eliminate light, which is present in the samples and capable of producing DO by autotrophes (algae). The BOD samples were incubated for five days, which was added to $2 \mathrm{ml}$ of each sample. In order to retard additional biological activities, Winkler solutions I and II use different dropping pipettes to each sample. The bottles were thoroughly shaken to precipitate the floc, which lay at the bottom of the bottles. Further, Winkler solution I is a solution of manganese sulphate, while solution II is sodium or potassium iodide, sodium or potassium hydroxide, sodium azide (sodium nitride) and sodium hydroxide. The DO samples were collected in clear bottles and also tightly stoppered. With samples of dissolved oxygen preserved on the spot with Winkler I and II solutions similar to that of the BOD samples (APHA, 2012). All samples had been clearly identified and controlled at $4^{\circ} \mathrm{C}$ for easy identification. Determination was carried out on site to know the concentrations of unstable as well as sensitive water quality characteristics including total dissolved solids (TDS), electrical conductivity (EC), $\mathrm{pH}$, alkalinity (Alka.), and temperature (Temp). Thus, the fundamental approaches for investigating the groundwater composition are described in figure 2 below.

\section{Quality assurance and quality control (QAVQC)}

Additionally, employing high purity analytical reagents as well as solvents, all analytical procedures were closely monitored with quality assurance as well as control techniques. Calibration standards were applied to the instruments. Procedure blanks, triplicate analysis as well as the analysis of certified reference materials (CRM) was performed through the analytical technique validation. For every organic 
pollutant from the groundwater samples, the limit of detection (LoD), repeatability, reproducibility, precision, as well as accuracy was established.

\section{Results}

Compare water quality parameters in the study areas with that of the recommended standards.

Table 3 \& 4: Descriptive statistics for each of the parameters and the recommended standards (Raining and Dry season) 
Table 3

Descriptive statistics for each of the parameters and the recommended standards (Raining season)

\begin{tabular}{|c|c|c|c|c|c|c|c|c|}
\hline SN & Parameters & Min. & Max. & Mean & SD & $\begin{array}{l}\text { WHO } \\
\text { standard }\end{array}$ & $\begin{array}{l}\text { SON } \\
\text { standard }\end{array}$ & $\begin{array}{l}\text { NAFDAC } \\
\text { standard }\end{array}$ \\
\hline 1. & $\begin{array}{l}\text { Temperature } \\
\left({ }^{\circ} \mathrm{C}\right)\end{array}$ & 24.00 & 32.40 & 27.83 & 2.00 & $15-30$ & Ambient & \\
\hline 2 & $\begin{array}{l}\text { Hydrogen } \\
\text { Potential (pH) }\end{array}$ & 3.57 & 8.98 & 6.59 & 1.11 & $6.5-8.5$ & $6.5-8.5$ & $6.5-8.5$ \\
\hline 3 & $\begin{array}{l}\text { Conductivity } \\
\left(\mathrm{YSCM}^{-1}\right)\end{array}$ & 10.55 & 89.91 & 31.83 & 20.22 & 300.00 & 1000.00 & 100 us/cm \\
\hline 4 & Turbidity (NTU) & 0.00 & 80.20 & 17.57 & 19.41 & 5.00 & $5 N T U$ & \\
\hline 5 & $\begin{array}{l}\text { Dissolved } \\
\text { Oxygen (DO) } \\
\text { (mg/l) }\end{array}$ & 15.90 & 19.65 & 16.92 & 0.98 & 7.50 & $>7.00$ & 6.00 \\
\hline 6 & $\begin{array}{l}\text { Biological } \\
\text { Oxygen } \\
\text { Demand (BOD) } \\
\text { (mg/l) }\end{array}$ & 4.87 & 6.09 & 5.28 & 0.35 & 5.00 & $<3.00$ & - \\
\hline 7 & $\begin{array}{l}\text { Chemical } \\
\text { Oxygen } \\
\text { Demand (COD) } \\
\text { (mg/l) }\end{array}$ & 22.20 & 51.00 & 36.60 & 9.60 & 10.00 & 10.00 & - \\
\hline 8 & Acidity (mg/l) & 56.30 & 169.56 & 91.17 & 41.54 & $\begin{array}{l}\text { No } \\
\text { Guidelines }\end{array}$ & & - \\
\hline 9 & Alkalinity (mg/l) & 14.79 & 165.16 & 110.91 & 61.15 & 100.00 & 200.00 & - \\
\hline 10 & $\begin{array}{l}\text { Total Hardness } \\
(\mathrm{TH})(\mathrm{mg} / \mathrm{l}) \\
(\mathrm{CaCO} 3)\end{array}$ & 30.00 & 52.00 & 39.17 & 5.83 & 100.00 & 100.00 & - \\
\hline 11 & $\begin{array}{l}\text { Total Dissolved } \\
\text { Solids (TDS) } \\
\text { (mg/l) }\end{array}$ & 5.16 & 16.18 & 9.57 & 4.11 & 250.00 & 500.00 & - \\
\hline 12 & $\begin{array}{l}\text { Total } \\
\text { Suspended } \\
\text { Solids (TSS) } \\
\text { (mg(l) }\end{array}$ & 29.80 & 42.09 & 37.05 & 3.03 & 3.00 & $>10.00$ & - \\
\hline 13 & Salinity (mg/l) & 0.00 & 30.00 & 6.12 & 10.29 & 600.00 & 500.00 & 600.00 \\
\hline 14 & Chloride (mg/l) & 25.48 & 33.51 & 30.60 & 2.09 & 200.00 & 100.00 & 100.00 \\
\hline 15 & Fluoride (mg/l) & 0.00 & 1.30 & 0.36 & 0.43 & $0.8-1.7$ & 1.00 & 1.50 \\
\hline 16 & $\begin{array}{l}\text { Aluminum } \\
(\mathrm{mg} / \mathrm{l})\end{array}$ & 0.00 & 0.04 & 0.01 & 0.01 & 0.20 & 0.02 & - \\
\hline
\end{tabular}




\begin{tabular}{|c|c|c|c|c|c|c|c|c|}
\hline SN & Parameters & Min. & Max. & Mean & SD & $\begin{array}{l}\text { WHO } \\
\text { standard }\end{array}$ & $\begin{array}{l}\text { SON } \\
\text { standard }\end{array}$ & $\begin{array}{l}\text { NAFDAC } \\
\text { standard }\end{array}$ \\
\hline 17 & $\begin{array}{l}\text { Sodium (Na) } \\
(\mathrm{mg} / \mathrm{l})\end{array}$ & 7.91 & 17.09 & 14.33 & 2.31 & 200.00 & 100.00 & - \\
\hline 18 & $\begin{array}{l}\text { Potassium (K) } \\
(\mathrm{mg} / \mathrm{l})\end{array}$ & 1.25 & 4.09 & 2.93 & 0.56 & 12.00 & 10.00 & - \\
\hline 19 & $\begin{array}{l}\text { Calcium (Ca) } \\
\text { (mg/l) }\end{array}$ & 40.37 & 64.33 & 54.80 & 7.77 & 75.00 & 75.00 & 75.00 \\
\hline 20 & $\begin{array}{l}\text { Magnesium } \\
(\mathrm{Mg})(\mathrm{mg} / \mathrm{l})\end{array}$ & 34.48 & 185.01 & 135.18 & 30.17 & 30.00 & 2.00 & 30.00 \\
\hline 21 & Iron (Fe) (mg/l) & 0.95 & 7.3 & 2.95 & 1.55 & 0.30 & 0.30 & 0.30 \\
\hline 22 & Zinc $(Z n)(m g / l)$ & 0.49 & 1.01 & 0.66 & 0.17 & 3.00 & 5.00 & 5.00 \\
\hline 23 & $\begin{array}{l}\text { Manganese } \\
(\mathrm{Mn})(\mathrm{mg} / \mathrm{l})\end{array}$ & 0.01 & 0.08 & 0.03 & 0.02 & 0.50 & 0.10 & 0.05 \\
\hline 24 & $\begin{array}{l}\text { Cadmium (Cd) } \\
(\mathrm{mg} / \mathrm{l})\end{array}$ & 0 & 0.07 & 0.01 & 0.02 & 0.003 & 0.003 & 0.003 \\
\hline 25 & $\begin{array}{l}\text { Lead }(\mathrm{Pb}) \\
(\mathrm{mg} / \mathrm{l})\end{array}$ & 0 & 0.19 & 0.11 & 0.06 & 0.01 & 0.01 & 0.01 \\
\hline 26 & $\begin{array}{l}\text { Copper (Cu) } \\
(\mathrm{mg} / \mathrm{l})\end{array}$ & 0.01 & 0.09 & 0.03 & 0.03 & 3.00 & 1.00 & 1.00 \\
\hline 27 & $\begin{array}{l}\text { Chromium (Cr) } \\
\text { (mg/l) }\end{array}$ & 0.05 & 2.84 & 0.87 & 0.89 & 0.05 & 0.01 & 0.05 \\
\hline 28 & $\begin{array}{l}\text { Sulphate }\left(\mathrm{SO}_{4}{ }^{2}\right) \\
(\mathrm{mg} / \mathrm{l})\end{array}$ & 0.67 & 1.31 & 0.87 & 0.14 & 250.00 & 100.00 & 100.00 \\
\hline 29 & $\begin{array}{l}\text { Ammonia } \\
\left(\mathrm{NH}_{3^{-}}\right)(\mathrm{mg} / \mathrm{l})\end{array}$ & 1.01 & 4.58 & 2.5 & 1.05 & $<0.20$ & 3.00 & - \\
\hline 30 & $\begin{array}{l}\text { Phosphate } \\
\left(\mathrm{PO}_{4}{ }^{3-}\right)(\mathrm{mg} / \mathrm{l})\end{array}$ & 0.08 & 0.59 & 0.22 & 0.16 & 0.10 & 0.30 & - \\
\hline 31 & $\begin{array}{l}\text { Nitrite }\left(\mathrm{NO}_{3}-\right) \\
(\mathrm{mg} / \mathrm{l})\end{array}$ & 0.65 & 4.67 & 1.79 & 1.14 & 45.00 & 0.002 & - \\
\hline 32 & $\begin{array}{l}\text { Nitrates }\left(\mathrm{NO}_{2}-\right) \\
(\mathrm{mg} / \mathrm{l})\end{array}$ & 1.64 & 5 & 2.45 & 0.89 & 50.00 & 10.00 & - \\
\hline 33 & $\begin{array}{l}\text { Nickel (Ni) } \\
(\mathrm{mg} / \mathrm{l})\end{array}$ & 0.61 & 2.36 & 0.92 & 0.29 & 0.02 & 0.02 & - \\
\hline 34 & $\begin{array}{l}\text { Total Petroleum } \\
\text { Hydrocarbon } \\
\text { (TPH) (mg/l) }\end{array}$ & 0 & 15 & 8.27 & 5.57 & $<10.00$ & $<10.00$ & - \\
\hline
\end{tabular}


Table 4

Descriptive statistics for each of the parameters and the recommended standards (Dry season)

\begin{tabular}{|c|c|c|c|c|c|c|c|c|}
\hline SN & Parameters & Min. & Max. & Mean & SD & $\begin{array}{l}\text { WHO } \\
\text { standard }\end{array}$ & $\begin{array}{l}\text { SON } \\
\text { standard }\end{array}$ & $\begin{array}{l}\text { NAFDAC } \\
\text { standard }\end{array}$ \\
\hline 1 & $\begin{array}{l}\text { Temperature } \\
\left({ }^{\circ} \mathrm{C}\right)\end{array}$ & 27.50 & 34.80 & 30.43 & 1.53 & $15-30$ & Ambient & \\
\hline 2 & $\begin{array}{l}\text { Hydrogen } \\
\text { Potential (pH) }\end{array}$ & 5.56 & 8.96 & 6.57 & 0.85 & $6.5-8.5$ & $6.5-8.5$ & $6.5-8.5$ \\
\hline 3 & $\begin{array}{l}\text { Conductivity } \\
\left(\mathrm{YSCM}^{-1}\right)\end{array}$ & 11.02 & 69.30 & 26.81 & 14.19 & 300.00 & 1000.00 & 100 us/cm \\
\hline 4 & $\begin{array}{l}\text { Turbidity } \\
\text { (NTU) }\end{array}$ & 1.71 & 52.00 & 6.56 & 9.34 & 5.00 & $5 N T U$ & \\
\hline 5 & $\begin{array}{l}\text { Dissolved } \\
\text { Oxygen (Do) } \\
\text { (mg/l) }\end{array}$ & 16.86 & 20.00 & 17.97 & 0.88 & 7.50 & $>7.00$ & 6.00 \\
\hline 6 & $\begin{array}{l}\text { Biological } \\
\text { Oxygen } \\
\text { Demand } \\
(B O D)(\mathrm{mg} / \mathrm{l})\end{array}$ & 4.75 & 6.13 & 5.57 & 0.46 & 5.00 & $<3.00$ & - \\
\hline 7 & $\begin{array}{l}\text { Chemical } \\
\text { Oxygen } \\
\text { Demand } \\
(\text { COD) }(\mathrm{mg} / \mathrm{l})\end{array}$ & 21.20 & 50.50 & 24.42 & 4.77 & 10.00 & 10.00 & - \\
\hline 8 & Acidity (mg/l) & 49.91 & 170.18 & 156.77 & 18.07 & $\begin{array}{l}\text { No } \\
\text { Guidelines }\end{array}$ & & - \\
\hline 9 & $\begin{array}{l}\text { Alkalinity } \\
(\mathrm{mg} / \mathrm{l})\end{array}$ & 14.59 & 135.70 & 44.40 & 25.89 & 100.00 & 200.00 & - \\
\hline 10 & $\begin{array}{l}\text { Total } \\
\text { Hardness (TH) } \\
(\mathrm{mg} / \mathrm{l}) \\
(\mathrm{CaCO} 3)\end{array}$ & 33.00 & 51.90 & 44.02 & 6.25 & 100.00 & 100.00 & - \\
\hline 11 & $\begin{array}{l}\text { Total } \\
\text { Dissolved } \\
\text { Solids (TDS) } \\
\text { (mg/l) }\end{array}$ & 5.40 & 16.71 & 12.34 & 4.29 & 250.00 & 500.00 & - \\
\hline 12 & $\begin{array}{l}\text { Total } \\
\text { Suspended } \\
\text { Solids (TSS) } \\
\text { (mg(l) }\end{array}$ & 28.60 & 35.50 & 33.27 & 1.84 & 3.00 & $>10.00$ & - \\
\hline 13 & Salinity $(\mathrm{mg} / \mathrm{l})$ & 0.00 & 30.00 & 4.14 & 8.02 & 600.00 & 500.00 & 600.00 \\
\hline 14 & $\begin{array}{l}\text { Chloride } \\
\text { (mg/l) }\end{array}$ & 24.81 & 31.61 & 28.21 & 2.34 & 200.00 & 100.00 & 100.00 \\
\hline
\end{tabular}




\begin{tabular}{|c|c|c|c|c|c|c|c|c|}
\hline SN & Parameters & Min. & Max. & Mean & SD & $\begin{array}{l}\text { WHO } \\
\text { standard }\end{array}$ & $\begin{array}{l}\text { SON } \\
\text { standard }\end{array}$ & $\begin{array}{l}\text { NAFDAC } \\
\text { standard }\end{array}$ \\
\hline 15 & Fluoride (mg/l) & 0.00 & 1.30 & 0.28 & 0.40 & $0.8-1.7$ & 1.00 & 1.50 \\
\hline 16 & $\begin{array}{l}\text { Aluminum } \\
(\mathrm{mg} / \mathrm{l})\end{array}$ & 0.00 & 0.04 & 0.01 & 0.01 & 0.20 & 0.02 & - \\
\hline 17 & $\begin{array}{l}\text { Sodium (Na) } \\
\text { (mg/l) }\end{array}$ & 8.06 & 13.44 & 11.39 & 1.80 & 200.00 & 100.00 & - \\
\hline 18 & $\begin{array}{l}\text { Potassium (K) } \\
(\mathrm{mg} / \mathrm{l})\end{array}$ & 1.17 & 4.15 & 2.82 & 1.03 & 12.00 & 10.00 & - \\
\hline 19 & $\begin{array}{l}\text { Calcium (Ca) } \\
\text { (mg/l) }\end{array}$ & 39.85 & 57.67 & 45.37 & 4.67 & 75.00 & 75.00 & 75.00 \\
\hline 20 & $\begin{array}{l}\text { Magnesium } \\
(\mathrm{Mg})(\mathrm{mg} / \mathrm{l})\end{array}$ & 131.01 & 185.01 & 174.61 & 13.78 & 30.00 & 2.00 & 30.00 \\
\hline 21 & $\begin{array}{l}\operatorname{Iron}(\mathrm{Fe}) \\
(\mathrm{mg} / \mathrm{l})\end{array}$ & 0.56 & 6.35 & 2.43 & 1.50 & 0.30 & 0.30 & 0.30 \\
\hline 22 & $\begin{array}{l}\operatorname{Zinc}(Z n) \\
(m g / l)\end{array}$ & 0.00 & 1.01 & 0.83 & 0.20 & 3.00 & 5.00 & 5.00 \\
\hline 23 & $\begin{array}{l}\text { Manganese } \\
(\mathrm{Mn})(\mathrm{mg} / \mathrm{l})\end{array}$ & 0.02 & 0.09 & 0.06 & 0.02 & 0.50 & 0.10 & 0.05 \\
\hline 24 & $\begin{array}{l}\text { Cadmium (Cd) } \\
(\mathrm{mg} / \mathrm{l})\end{array}$ & 0.00 & 0.07 & 0.03 & 0.02 & 0.003 & 0.003 & 0.003 \\
\hline 25 & $\begin{array}{l}\text { Lead (Pb) } \\
(\mathrm{mg} / \mathrm{l})\end{array}$ & 0.00 & 0.15 & 0.01 & 0.02 & 0.01 & 0.01 & 0.01 \\
\hline 26 & $\begin{array}{l}\text { Copper (Cu) } \\
(\mathrm{mg} / \mathrm{l})\end{array}$ & 0.06 & 0.10 & 0.08 & 0.01 & 3.00 & 1.00 & 1.00 \\
\hline 27 & $\begin{array}{l}\text { Chromium (Cr) } \\
(\mathrm{mg} / \mathrm{l})\end{array}$ & 0.01 & 2.85 & 2.03 & 0.93 & 0.05 & 0.01 & 0.05 \\
\hline 28 & $\begin{array}{l}\text { Sulphate } \\
\left(\mathrm{SO}_{4}{ }^{2}\right)(\mathrm{mg} / \mathrm{l})\end{array}$ & 0.80 & 1.28 & 1.01 & 0.13 & 250.00 & 100.00 & 100.00 \\
\hline 29 & $\begin{array}{l}\text { Ammonia } \\
\left(\mathrm{NH}_{3^{-}}\right)(\mathrm{mg} / \mathrm{l})\end{array}$ & 1.00 & 4.66 & 3.71 & 1.10 & $<0.20$ & 3.00 & - \\
\hline 30 & $\begin{array}{l}\text { Phosphate } \\
\left(\mathrm{PO}_{4}{ }^{3-}\right)(\mathrm{mg} / \mathrm{l})\end{array}$ & 0.30 & 0.60 & 0.44 & 0.07 & 0.10 & 0.30 & - \\
\hline 31 & $\begin{array}{l}\text { Nitrite }\left(\mathrm{NO}_{3}-\right) \\
(\mathrm{mg} / \mathrm{l})\end{array}$ & 1.01 & 4.65 & 3.30 & 0.86 & 45.00 & 0.002 & - \\
\hline 32 & $\begin{array}{l}\text { Nitrates }\left(\mathrm{NO}_{2}-\right) \\
(\mathrm{mg} / \mathrm{l})\end{array}$ & 1.19 & 4.91 & 3.29 & 1.02 & 50.00 & 10.00 & - \\
\hline
\end{tabular}




\begin{tabular}{|clccccccc|}
\hline SN & Parameters & Min. & Max. & Mean & SD & $\begin{array}{l}\text { WHO } \\
\text { standard }\end{array}$ & $\begin{array}{l}\text { SON } \\
\text { standard }\end{array}$ & $\begin{array}{l}\text { NAFDAC } \\
\text { standard }\end{array}$ \\
\hline 33 & $\begin{array}{l}\text { Nickel (Ni) } \\
\text { (mg/l) }\end{array}$ & 0.72 & 2.56 & 1.21 & 0.25 & 0.02 & 0.02 & - \\
\hline 34 & 0.00 & 15.00 & 6.34 & 5.39 & $<10.00$ & $<10.00$ & - \\
\hline & $\begin{array}{l}\text { Total } \\
\begin{array}{l}\text { Petroleum } \\
\text { Hydrocarbon } \\
\text { (TPH) (mg/l) }\end{array}\end{array}$ & & & & & & \\
\hline
\end{tabular}

Table 3 and Table 4 present comparison result of the water quality characteristics obtained in the study location during wet and dry season with that provided by WHO, SON and NAFDAC recommended standards. During wet season result indicates that $\mathrm{pH}$, temperature and TPH were within the recommended standards while alkalinity was within SON standard but above WHO standard. Result also reveals that other water quality parameters were either below or above the recommended standards as provided by WHO, SON and NAFDAC with mean values of conductivity, total hardness, TDS, salinity, chloride, fluoride, aluminum, $\mathrm{Na}, \mathrm{Zn}, \mathrm{Mn}, \mathrm{Cu}, \mathrm{SO}_{4}, \mathrm{NO}_{3}, \mathrm{NO}_{2}, \mathrm{~K}$ and $\mathrm{Ca}$ were below the standard while other parameters values were above the recommended standards. For dry season, the mean value for $\mathrm{Pb}, \mathrm{pH}$, and TPH were within standard while that of conductivity, alkalinity, total hardness, TDS, salinity, $\mathrm{Cl}, \mathrm{F}, \mathrm{Al}$, $\mathrm{Na}, \mathrm{Zn}, \mathrm{Mn}, \mathrm{K}, \mathrm{Ca}, \mathrm{Cu}, \mathrm{SO}_{4}, \mathrm{NO}_{3}{ }^{-}$and $\mathrm{NO}_{2}{ }^{-}$were below the recommended standards while other water quality parameters obtained during dry season were above the standards as provided in Table 4 .

\section{Discussion}

Compare water quality parameters in the study areas with that of the recommended standards. [Groundwater Suitability for Drinking and Irrigation]

It is essential to determine the appropriateness of the groundwater resources in a researched area, since it is an essential element. Tables 3 and 4 consolidate the descriptive findings of WHO, SON and NAFDAC water quality parameters collected in the field in wet and dry seasons with those of the recommended criteria. On a background of highly fluctuating physicochemical composition, the results presented in this study must remain interpreted prudently on a case-by-case basis. As the "tragedy of open access" of the groundwater resource in Ebocha-Obrikom area of Rivers State was unfolding, public debate centered on two questions: a) what is the true extent of groundwater contamination? and b) what are the sources of contamination? In the Niger Delta regions, groundwater quality assessments are essential toward facilitating sustained economic as well as social development, including human survival. In this study, groundwater quality spatial distribution was examined in Ebocha-Obrikom area of Rivers State, while addressing the mechanisms governing groundwater chemistry. For all sorts of life on earth, water been the most important resources. The drinking water quality undoubtedly impacts the lives of indigenous people. The intake or use of polluted/contaminated water is the main cause of human illnesses that has produced a major health problem (Raimi et al., 2017; Morufu and Clinton, 2017; Raimi and Sabinus, 2017; Raimi et al., 2018; Olalekan et al., 2018; Raimi et al., 2019; Olalekan et al., 2019; Raimi et al., 2019; 
Suleiman et al., 2019; Gift and Olalekan, 2020; Gift et al., 2020; Olalekan et al., 2020; Afolabi and Raimi, 2021). Table 3 and 4 show the physico-chemical and heavy-metal findings of groundwater samples. These findings are compared to the prescribed guideline by WHO, SON and NAFDAC Standards. After having compared the finding with these standards, it becomes obvious that chemical oxygen demand (COD), turbidity, biological oxygen demand (BOD), dissolved oxygen (DO), alkalinity, magnesium (Mg), Iron, Cadmium, Lead, total suspended solids (TSS), Chromium, Ammonia, Phosphate, Nitrite and Nickel are major worrying factors in Ebocha-Obrikom area of Rivers State. Other physico-chemical parameters reveal that other water quality parameters were either below or within the recommended standards as provided by WHO, SON and NAFDAC in most sites or display values exceedingly be found at isolated pockets in some sites. Therefore, other parameters analyzed remain not remarkable in discussion. Ebocha-Obrikom groundwater quality remained worst affected. In the current investigation, the drinking water temperature varied from 27.83 (rainy) to $30.43^{\circ} \mathrm{C}$ (dry). One of the essential physicochemical parameters is water temperature, since it changes both temporally as well as geographically. It impacts the water capacity toward holding oxygen as well as aquatic organism's metabolic rates. It likewise, impacts trace metals availability that have an indirect influence on water $\mathrm{pH}$. It impacts fish reproductive behavior as well as growth. With a rise in temperature, the solids solubility increases as the gases decrease. Significant aquatic life gases therefore diminish with temperature increase. Temperature generally influences the fundamental rate of biochemical processes, therefore regulating attributes of the organism together with development rate as well as survival.

Temperature of water influences the biological reactions in water. Higher values of temperature accelerated the chemical reaction in water. Thus, allaying the conceivable fear of thermal shocks on the receiving ground water bodies. The groundwater capacity toward reacting with geological materials is determined by the $\mathrm{pH}$ value when controlling ions existing in groundwater (Islam et al. 2017; Morufu and Clinton, 2017; Raimi and Sabinus, 2017; Olalekan et al., 2018). The pH is remarkably vital for understanding water nature, as well as likewise make evident a tight proximity with other water chemical components. The value of $\mathrm{pH}$ in drinking water is a significant index of alkalinity, acidity and resulting value of acid base interaction of its minerals and organic components. Measurement of $\mathrm{pH}$ is one of the most essential recurrently used water chemistry test. Although the $\mathrm{pH}$ is not directly affecting human health, it is one of the utmost significant parameters for water quality. A suitable pH range of 6.5 to 8.5 is generally adopted, according to the $\mathrm{WHO}$ recommendation. $\mathrm{pH}$ values observed in the range of 6.57 to 6.59 in the water samples were found in this study. This demonstrates that $\mathrm{pH}$ was perceived to be slightly demonstrating naturally acidic. From the groundwater results, the $\mathrm{pH}$ of the water samples fell within the recommended standards as provided by WHO, SON and NAFDAC during wet and dry season. In any of the groundwater testing, however, both seasons were found to remain within the maximum allowed limit. As a result, based on $\mathrm{pH}$ as an indication of agriculture and population, the groundwater seems not to appear to be a serious pollution threat to the milieu. Thus, the $\mathrm{pH}$ water value of the test zone is clearly below the allowed limit (6.5-8.5). There is no misunderstanding, therefore, that the region is neither overly acidic or alkaline. Turbidity denotes the relative purity of the water which hinders light transmission and is triggered by chemicals that do not occur in true solution form as well as is directly 
connected to light dispersal. The ground water turbidity in the research area shows higher turbidity during rainy season $(17.57 \mathrm{mg} / \mathrm{L})$ representing higher rate of light scattering affecting photosynthesis. Thus, higher turbidities could be due to continuous and impactful predisposition to receiving large quantities of organic and inorganic materials emanating from gas flaring and oil spillage contaminating the ground waters of the study area. Thus, the maximum turbidity level surpassed the WHO/NAFDAC/SON drinking water quality recommendations. It is therefore doubtful that it will be appropriate for drinking. As numerous previous studies have revealed that the turbidity in Ebocha-Obrikom groundwater area is significantly more than the permissible levels of the WHO/NAFDAC. Likewise, dissolved oxygen (DO) is the oxygen gas $\left(\mathrm{O}_{2}\right)$ that is dissolved in water and it is a highly significant water indicator/constituents which affect physiological and biological process and for its quality as well as help to maintain biological life in aquatic ecosystem. For excellent water quality, enough dissolved oxygen is required. All kinds of life are made out of oxygen. Oxygen influences a large range of other water indicators, such as the odour, clarity as well as taste not only biochemical, but also aesthetic ones. Oxygen removes from the water through aquatic living things respiration as well as organic matter decomposition. A higher metabolic activity as well as inorganic reducing agents like $\mathrm{NH}_{3}, \mathrm{H}_{2} \mathrm{~S}, \mathrm{NO}_{2}$ as well as $\mathrm{Fe}^{++}$ions have reduced water oxygen balance (Raimi and Sabinus, 2017; Morufu and Clinton, 2017; Olalekan et al., 2018). The oxygen maxima was observed during rainy season (16.92mg/L) suggesting lower amount of inorganic reducing agents during this present research. Organic matter decomposition may remain a remarkable factor toward reducing dissolved oxygen during dry season $(17.97 \mathrm{mg} / \mathrm{L})$. Thus, the low dissolved oxygen levels are probably due to the biological oxygen demand associated with cycling of bacteria and fungi in the ground water (Olalekan et al., 2018; Henry et al., 2019; Afolabi and Raimi, 2021) and the large quantity of biodegradable organic compounds in groundwater. Likewise, Malcolm et al., (2003) report that groundwater upwelling reduces the salmonid eggs survival rate owing to its low dissolved concentrations of oxygen as well as its chemically reduced properties. This demonstrate both the flux as well as groundwater chemical composition as a controlling element for the hyporheic habitat. This indicates that inorganic dissolved minerals $\left(\mathrm{S}, \mathrm{NO}_{3}, \mathrm{Ca}\right)$ do influence water purity as well as making it more poisonous. Therefore, it remains deduced that larger rates of human activities and seasonal fluctuation seem to influence dissolved oxygen, as higher DO signify biological activity which is a reflection of high organic matter input. Low DO levels cause stress and anaerobic decomposition of organic matter. These results corroborate earlier report by Olalekan et al., (2018) that high or low values impact aquatic life and change in one form or another the toxicity of other pollutants. DO in liquid offers an oxygen supply needed to oxidize organic materials if its concentration is high as well as deficiency of it causes body of water to become lifeless or devoid of aquatic life (Morrison et al., 2001; Chukwu, 2008). This result found support in Dami, Ayuba and Amukali (2012) which established that ground water may likewise remain conveniently used toward supporting and sustaining fishing pond operations since the values of DO were above the required value of greater than 7 as the fishing stream standard. In most water quality studies, USEPA (2001) states that the DO is a metric and is proposed by the EPA as an added prime response variable for systems that have previously experienced hypoxia. Biological oxygen demand (BOD) is the quantity of dissolved oxygen (DO) necessary to stabilize the biodegradable organic matter through micro-organisms of water 
under aerobic conditions. It likewise comprises the oxygen essential for the oxidation of several chemical in the water like iron, sulfides, ferrous as well as ammonia (Olalekan et al., 2018; Adewumi and Laniyan, 2020; Onyemesili et al., 2020; Afolabi and Raimi, 2021). Higher BOD values in the dry season may attribute to the stagnations of water body leading to the absence of self- purification cycle. Thus, gas flaring must have contributed to this trend whereby the highest values between raining and dry seasons were observed. This tend to supports higher biochemical activity. As anthropogenic activities could influence higher BOD just as seasonality influences BOD. This parameter hence, offers information on the microbial respiration potential to breakdown the organic matter that might contribute to low DO in water and is a proposed cause of hypoxia (Olalekan et al., 2018; Ezenwaji and Ezenweami, 2018; Egbueri and Unigwe, 2020; Afolabi and Raimi, 2021). The need with respect to chemical oxygen demand (COD) is the quantity of oxygen needed by use of a strong oxidant as well as to convert it to carbon dioxide and water toward oxidizing organic matter in waste water (Morufu and Clinton, 2017; Raimi and Sabinus, 2017; Olalekan et al., 2018; Afolabi and Raimi, 2021). To evaluate the pollution degree in the area under investigation, $C O D$ test is employed. COD value is constantly greater than that of $\mathrm{BOD}_{5}$ since several organic substances can remain oxidized chemically but not biologically (Morufu and Clinton, 2017; Raimi and Sabinus, 2017; Olalekan et al., 2018; Afolabi and Raimi, 2021). Thus, chemical oxygen demand (COD) values was found to be highest during the raining season with an average value of $(36.60) \mathrm{mg} / \mathrm{L}$ followed by $24.42 \mathrm{mg} / \mathrm{L}$ during the dry season. All recorded values in the study area were above the maximum permissible limit set for COD by WHO/SON/NAFDAC. Thus, it could be deduced that areas prone to oil related activities as gas flaring and oil spillage influence higher COD more than areas that are not under the influences of such activities. Since COD measure oxygen demand created by organic and inorganic compounds as well as by biodegradable compounds. The trend shows that higher chemical activity could be attributed to gas flaring and oil spill impact. Hence, increase of chemical oxygen demand (COD) values are due to the pollution of input Zones. As it could be established that raining seasons influence COD more than its dry counterpart. Thus. the higher the BOD (or COD), the higher the pollution degree (Morufu and Clinton, 2017; Raimi and Sabinus, 2017; Olalekan et al., 2018; Uhah et al., 2019; Afolabi and Raimi, 2021). Alkalinity is a water neutralizing capability measure of acids or ions of hydrogen. If there are variations made to the $\mathrm{pH}$ value of water's, Alkalinity acts as a buffer. Alkalinity will contribute to stabilizing the $\mathrm{pH}$ of the water. A neutral $\mathrm{pH}$ of 7 is required for drinking water. It is beneficial to have high alkalinity in our drinking water since it safeguards the water for us to consum. The amount of alkalinity that should be in our water is $100-200 \mathrm{mg} / \mathrm{l}$ for typical drinking water. Alkalinity is basically dissolved minerals in the water that help neutralize the water we drink. Thus, higher values of alkalinity during rainy season (110.91) were due to leaching of soil during natural filtration of water from sewage. The alkalinity is primarily due to bicarbonate ions largely caused by bacterial breakdown of organic mineral along with exchange of mineral ion which varies from (44.40-110.91 mg/L) in study area. The water samples for rainy season was observed $(110.91 \mathrm{mg} / \mathrm{L})$ at rainy season having more value of alkalinity than the WHO tolerable limit in the research location. Thus, bicarbonate and hydroxides can be attributable to alkalinity in natural waters. The consequential effects of water eutrophication are affected by alkalinity (USEPA, 2019). For Total Suspended Solid (TSS), the mean values of TSS show that the highest value was observed during the raining season $(37.05) \mathrm{mg} / \mathrm{L}$. While, the least value was recorded 
during the dry season (33.27) $\mathrm{mg} / \mathrm{L}$. All values recorded in this study were above the recommended maximum permissible limit. Generally, it could be deduced that seasons affected TSS more than the level of anthropogenic activities within the study area. This is because increased atmospheric precipitation during raining seasons help increases the amount of materials in ground waters unlike during the dry seasons where reduced precipitation help in removing suspended particles in groundwater. Also, excessive influx of suspended solids during the raining season could be attributed to discharge of large quantities of substances directly into surface water bodies or out rightly onto terrestrial regions from where they percolate into ground water bodies. This could be owing to the fact that gas flaring releases persistent non-combustible chemicals while oil spillage releases less dense volatile chemicals into the atmosphere where they condense and later fall back as rain to the groundwater. Magnesium had comparatively high values during the dry season $(174.61) \mathrm{mg} / \mathrm{L}$ compared to the raining season (135.18) $\mathrm{mg} / \mathrm{L}$. In this investigation, all values documented were above the WHO/SON/NAFDAC maximum acceptable limit. Higher values of magnesium above the maximum permissible value can be ascribed to rock weathering, which disintegrates chemical elements such as magnesium into surface water bodies from where it percolates into ground water bodies. This chemical weathering could be affected by a number of factors, hence the non-uniform variations between seasons. Also, it could be deduced that seasonal variations have significant influence upon the concentration of magnesium within the study area. The findings are similar with Olalekan et al., (2018). It may be concluded that the drinking water supply for Ebocha-Obrikom area of Rivers State is not entirely safe and a filtration plant has to be installed in Ebocha-Obrikom immediately on the basis of physico-chemical properties.

While, trace metal may be hazardous to human health and has a tremendous health impacts even if they are ingested in excess as well as accrues in human bodies at low concentrations (Raimi et al., 2017; Morufu and Clinton, 2017; Raimi and Sabinus, 2017; Olalekan et al., 2018; Raimi, 2019; Olalekan et al., 2020; Afolabi and Raimi, 2021). Even though, trace elements are rare but can be incredibly important to the oceans. Some of these elements are helping scientists understand the oceans' role in changing climate, past and the present, which can help predict climate in the future (Raimi et al., 2018; Raimi et al., 2019; Morufu et al., 2021). While, iron in groundwater causes a "rusty" taste in water. It not only gives an unpleasant taste; it can also stain pipes as well as clothing. Iron is natural in nature, and it is the fourth rich earth element in ground water of the research area occurring cosmopolitically, and thus most groundwater has a certain quantity of iron in it. Iron is derived from the earth minerals, like shale, limestone, as well as coal. In this study, the concentration of iron content for raining seasons ranges from 0.95 to $7.3 \mathrm{mg} / \mathrm{L}$ with a mean of $2.95 \mathrm{mg} / \mathrm{L}$, and for dry seasons, Fe ranges from 0.56 to $6.35 \mathrm{mg} / \mathrm{L}$ with a mean of $2.43 \mathrm{mg} / \mathrm{L}$, Fe concentration exceeds the allowable drinking purpose limit. While iron deficiency causes hemoglobin as well as cytochromes to decreases, excess of it causes tissues damage through iron accumulation. Hence, iron was found exceeding maximum permissible recommended limits provided by WHO, SON and NAFDAC. It is evident in general, the Ebocha-Obrikom ground water bodies contain higher amounts of iron as well as make the water inappropriate in a range of domestic/ residential purposes. Many treatment options are available, among them, water softener installation. In iron precipitation, aeration, oxygen addition to the water can also help to eliminate it from the water. 
Besides, Iron was also the second richest metal on the earth's crust in the world (USEPA, 2017). Iron is the $26^{\text {th }}$ elemental position in the periodic table as well as constitutes the key element to nearly all living creatures' growth and survival (Valko et al., 2005; Olalekan et al., 2018; Olalekan et al., 2020; Afolabi and Raimi, 2021). This is an essential component of organisms such as algae as well as enzymes like cytochromes as well as catalase, and proteins that transport oxygen, like hemoglobin and myoglobin (Vuori, 1995; Olalekan et al., 2018; Olalekan et al., 2020; Afolabi and Raimi, 2021). Iron, due to its interconversion between the ferrous $\left(\mathrm{Fe}^{2+}\right)$ as well as ferric $\left(\mathrm{Fe}^{3+}\right)$ ions is the attractive transition metal for a variety of redox biological processes (Phippen et al., 2008; Olalekan et al., 2018; Olalekan et al., 2020). The iron source is anthropogenic in ground water as well as is associated with mining as well as flaring activities. Sulfuric acid production as well as ferrous $\left(\mathrm{Fe}^{2+}\right)$ discharge is due to iron pyrites $\left(\mathrm{FeS}_{2}\right)$ oxidation which are prevalent in coal seams (Valko et al., 2005; Olalekan et al., 2018; Olalekan et al., 2020). The equations below epitomize the simplified ferrous oxidation reaction as well as ferric iron (Phippen et al., 2008; Olalekan et al., 2018; Olalekan et al., 2020):

$$
2 \mathrm{FeS}_{2}+7 \mathrm{O}_{2}{ }^{\circledR} 2 \mathrm{FeSO}_{4}+\mathrm{H}_{2} \mathrm{SO}_{4} \text { (ferrous) }
$$

$4 \mathrm{FeSO}_{4}+\mathrm{O}_{2}+10 \mathrm{H}_{2} \mathrm{O} \circledast 4 \mathrm{Fe}(\mathrm{OH})_{3}+4 \mathrm{H}_{2} \mathrm{SO}_{4}$ (ferric)

The dissolved iron concentration is typically $0.6 \mathrm{nM}$ or $33.5 \times 10-9 \mathrm{mg} / \mathrm{L}$ in deep ocean, as well as in freshwater, the detection level concentration is extremely low with $5 \mu \mathrm{g} / \mathrm{L}-\mathrm{ICP}$, however in groundwater, the dissolved iron concentration is rather high with $20 \mathrm{mg} / \mathrm{L}$ (USEPA, 2017). Several people in the Niger Delta region of Nigeria were exposed to higher concentrations of iron by means of drinking water, as the groundwater collected exceeded the WHO/SON/NAFDAC acceptable limit for drinking water quality (Olalekan et al., 2018; Afolabi and Raimi, 2021). The species abundance, including benthic invertebrates, periphyton as well as fish diversity are severely influenced by the direct as well as indirect iron contamination effects (Vuori, 1995). The precipitate iron will cause significant harm through clogging action as well as hinder fishes' respiration (USEPA, 2017). Lead (Pb), and cadmium (Cd) are highly toxic metals that have no known physiological role but can cause adverse health effects even at trace levels. The higher risk of high blood pressure (hypertension) as well as cardiovascular disease (CVD) in adults is associated to exposure to certain metals (Chowdhury et al., 2018; Hu et al., 2018; Lanphear et al., 2018; Olalekan et al., 2020) and in pregnant women (Liu et al., 2019). The prevalence of exposure to these metals among pregnant women is high. The general resident of Ebocha-Obrikom is ubiquitously exposed to toxic metal such as cadmium through the diet and gas flaring as the main sources. Cadmium exposure is associated with increased morbidity and mortality in myocardial infarction and stroke. Atherosclerosis is the main underlying mechanism of myocardial infarction. However, associations between cadmium and coronary artery atherosclerosis have not been examined. Cadmium accumulates mainly in the kidneys and has a half-life of decades, and therefore it usually increases with age. Exposure and body burden can be assessed by measuring blood or urine cadmium concentrations (Nordberg et al., 2015; Akerstrom et al., 2013). Apart from being a well-known cause of kidney and skeletal damage, blood or urine, cadmium has been reported to be an independent cardiovascular disease risk factor, together with 
coronary heart disease in several reviews (Tellez-Plaza et al., 2013a; Chowdhury et al., 2018; Olalekan et al., 2018; Tinkov et al., 2018; Olalekan et al., 2020). Though, cadmium is a $20^{\text {th }}$ century metal. It is a byproduct of the production of zinc. Soils as well as rocks comprise a certain quantity of cadmium, together with coal as well as mineral fertilizers. Cadmium is frequently utilized in electroplating and in various applications, for example in batteries, plastics, pigments as well as metal coatings (Martin \& Griswold, 2009; Olalekan et al., 2018; Koleayo et al., 2021).

The International Agency for Research on Cancer classifies cadmium and its compounds as Group 1 carcinogenic for humans. Natural processes like volcanic eruptions, river transport, weathering, as well as some human activities like mining, gas flaring, tobacco smoking, smelting, incineration of municipal waste, and fertilizers production released cadmium into the milieu. Even though in most developed nations, cadmium emissions have been substantially decreased, it remains a cause of worry for employees and individuals who live in the contaminated areas. Cadmium can produce both acute as well as chronic intoxications (Chakraborty et al., 2013). Cadmium is very harmful to the kidney as well as it accumulates in increasing concentrations in the proximal tubular cells. Cadmium can induce bone mineralization either as a result of a bone damage or as a result of renal failure. Humans as well as animals' studies have indicated that osteoporosis (skeletal deterioration) is a key consequence of cadmium exposure coupled with calcium metabolism disturbances, renal stones formation as well as hypercalciuria. Inhaling larger quantities of cadmium can lead to serious harm to the lungs. Ingested cadmium in greater amounts can cause stomach discomfort as well as diarrhea and vomiting. On extremely long exposure duration at lower concentrations, it can get deposited in the kidney as well as finally cause fragile bones, kidney disease, as well as lung damage (Bernard, 2008). In comparison to other metals, cadmium and its derivatives are extremely water soluble. Because of their high bioavailability, they tend to bioaccumulate in the body. Long-term cadmium exposure can cause morphopathological abnormalities in the kidneys. Cadmium poisoning is more common in smokers than in nonsmokers. Tobacco plants, like other plants, may acquire cadmium from the soil, making it the principal source of cadmium intake in smokers. Cadmium is ingested by non-smokers through gas flaring, food as well as some other sources. However, cadmium uptake via other routes is significantly much lower (Mudgal et al., 2010). Cadmium toxicity effects are caused by interactions with vital nutrients. $50 \%$ of cadmium is absorbed in the lungs as well as less in the gastrointestinal tract, according to experimental animal analysis. If cadmium exposure is significantly high during human pregnancy, it can lead to problems such as premature birth as well as reduced birth weights are the issues that can occur (Olalekan et al., 2018; Olalekan et al., 2020). Groundwaters under study are not potable in relation to the concentrations of $\mathrm{Cd}, \mathrm{Pb}$ as well as Fe. The borehole water and well water from Ebocha-Obrikom area also contains high lead concentration, particularly during rainy season. Though iron is not regarded a health issue in small amounts, it is nonetheless considered a nuisance when consumed in large amounts. Long-term consumption of drinking water containing a high concentration of iron can result in liver damage. Laundry staining as well as ceramic ware occurs when iron levels is above $0.30 \mathrm{mg} / \mathrm{L}$. When present in concentration greater than $1.0 \mathrm{mg} / \mathrm{L}$, dissolved iron in drinking water is difficult to remove. The high content iron in groundwater in the Ebocha-Obrikom area of Rivers State, can be linked 
to two factors: first, the bedrock of Ebocha-Obrikom area of Rivers State is fluvial sediments, which also comprises iron as well as the second - probable leaching and fall out from gas flaring and sponge iron plants- as the outcome shows areas nearby gas flaring plants and sponge iron plant has high iron content. Lead as well as its derivatives remain toxic to all living things. As a cumulative poison, it tends to lodge in the bones and can disrupt or lead to chromosome damage. Brain damage, anaemia, brain tumors, cancer, neonatal defects, central as well as peripheral nervous system collapse have all been linked to Lead $(\mathrm{Pb})$. The lead average concentration in current ground water samples is greater than 0.01 $\mathrm{mg} / \mathrm{L}$ (ranging between $0.11 \mathrm{mg} / \mathrm{L}$ in the rainy season and $0.01 \mathrm{mg} / \mathrm{L}$ in the dry season), the value of the rainy season is above the $0.01 \mathrm{mg} / \mathrm{L}$ standard of the drinking water quality. This outcome suggests that the source of increasing $\mathrm{Pb}$ concentration could remain due to the activities of the oil and gas industries and some other sources. Apparently, long term lead accumulation from gas flaring emissions is most likely the source of high $\mathrm{Pb}$ levels in water of the study area. While, lead on the other hand is a very poisonous metal whose widespread usage has resulted in significant extensive environmental pollution as well as created health concerns in several regions of the global south. In a dry atmosphere, lead is a bright silvery metal that is slightly bluish. When it comes into contact with air, it begins to tarnish, thereby generating a complicated compounds mixture that varies according to given circumstances. Industrial developments, food as well as smoking, domestic sources and drinking water remain the main lead source exposure. Gasoline as well as house painting were the main lead sources, which was later extended toward lead bullets, toys, pewter pitchers, storage batteries, plumbing pipes as well as faucets (Olalekan et al., 2018; Olalekan et al., 2020). Some of it is taken up via plants, soil fixation as well as released into water bodies, so exposure by human to lead in the overall population is either through food or drinking water (Goyer, 1990; Raimi et al., 2021; Olalekan et al., 2021). Lead is a highly poisonous heavy metal that interferes with a variety of plant physiological systems as well as unlike other trace metals like copper, zinc as well as manganese, it has no biological activities. A plant with a high concentration of lead produces more reactive oxygen species (ROS), which damages the lipid membrane that eventually leads to chlorophyll damage as well as photosynthetic processes and suppressing the plant general growth (Okoyen et al., 2020; Raimi et al., 2021; Olalekan et al., 2021). Some enquiry discovered that lead is capable of suppressing tea plant growth via reducing biomass as well as debases the quality of tea through altering the components quality (Yongsheng et al., 2011). Treatment of lead was discovered to induce significant ion uptake instability in plants, which leads to remarkable metabolic alterations in photosynthetic capacity as well as eventually, a strong suppression of plant development, even at low concentrations (Mostafa et al., 2017). The element chromium being the seventh most abundant on the planet, in the environment, chromium occurs in a variety of oxidation states, ranging from $\mathrm{Cr}^{2+}$ to $\mathrm{Cr}^{6+}$ (Rodríguez et al., 2007; Mohanty \& Kumar Patra, 2013). Trivalent- $\mathrm{Cr}^{+3}$ and hexavalent $\mathrm{Cr}^{+6}$, are the two most frequent forms of $\mathrm{Cr}$, as well as both are harmful to animals, people as well as plants (Mohanty and Kumar-Patra, 2013). Oil as well as coal burning, petroleum from ferro chromate refractory material, catalyst, pigment oxidants, fertilizers, chromium steel, oil well drilling as well as metal plating tanneries are all-natural sources of chromium. Chromium is discharged into the milieu anthropogenically through sewage as well as fertilizers (Ghani, 2011). $\mathrm{Cr}$ (III) is immobile and insoluble in water in its reduced form, but $\mathrm{Cr}(\mathrm{VI})$ is extremely soluble in water as well as consequently mobile in its oxidized state (Wolińska et 
al., 2013). Speciation of metal is critical for determining the metal ions activities in the milieu, as the oxidative form of $\mathrm{Cr}(\mathrm{III})$ is not a crucial ground water contaminant in the case of chromium, but $\mathrm{Cr}(\mathrm{VI})$ has been recognized to be toxic for humans. $\mathrm{Cr}(\mathrm{III})$ resides in the organic matter of soil as well as aquatic environment in oxides form, hydroxides as well as sulphates (Cervantes et al., 2001; Cervantes et al., 2017; Raimi et al., 2021). Chromium is widely utilized in industries like tanning, electroplating, metallurgy, production of paints and pigments, chemical and petroleum production, wood preservation, pulp as well as production of paper. These industries contribute significantly to chromium pollution, which has a negative impact for biological as well as ecological species (Ghani, 2011; Raimi et al., 2021; Olalekan et al., 2021). Chromium pollution is a concern due to a variety of industrial as well as agricultural practices that raise the harmful levels in the milieu. In recent years, chromium pollution of the milieu, mostly hexavalent chromium, has become a major concern (Zayed \& Terry, 2003; Olalekan et al., 2018; Olalekan et al., 2020). Several harmful heavy metals and chemicals are released into water streams by tanneries (Nath et al., 2008; Odipe et al., 2018). Cr (III) is oxidized to $\mathrm{Cr}(\mathrm{VI})$ in the presence of sufficient oxygen in the environment, which is exceedingly poisonous and highly water soluble (Cervantes et al., 2001; Cervantes et al., 2017). The concentration of chromium in soil has risen dramatically as a result of industrial waste discharge and ground water contamination (Olalekan et al., 2021). The deposit of $\mathrm{Cr}$ residues as well as waste water irrigation during chromate manufacturing constituted a major $\mathrm{Cr}$ pollution threat to farmland. As a result of the implementation of modern agriculture, there is a constant release of $\mathrm{Cr}$ into the milieu through $\mathrm{Cr}$ residues, $\mathrm{Cr}$ dust, as well as $\mathrm{Cr}$ waste water irrigation, resulting in soil pollution that affects the soil-vegetable system as well as disrupts vegetable yield and quality for humans (Duan et al., 2010; Raimi and Sabinus, 2017). Excess chromium in the environment is harmful to plants because it alters the biological aspects of the plant and enters the food chain through the eating of these plant materials (Raimi et al., 2021; Olalekan et al., 2021). Reduced root growth, seed germination inhibition, leaf chlorosis as well as decreased biomass remain common symptoms of Cr phytotoxicity. Chromium toxicity has a significant impact on biological processes in several plants like wheat, maize, cauliflower, barley, citrullus as well as in vegetables (Raimi and Sabinus, 2017). Chromium toxicity in plants causes chlorosis and necrosis (Ghani, 2011). Chromium toxicity affects enzymes containing iron as a component such as catalase, peroxidase as well as cytochrome oxidase. For natural ammonia levels in groundwater are typically less than $0.2 \mathrm{mg} / \mathrm{I}$ (WHO, 2017). Increased concentrations, on the other hand, are a sign of bacterial, sewage, as well as animal waste pollution (Sawyerr et al., 2018; Raimi et al., 2021; Olalekan et al., 2021). Ammonia levels in this study ranged from $2.5 \mathrm{mg} / \mathrm{l}$ during the rainy season to 3.71 $\mathrm{mg} / \mathrm{l}$ during the dry season. Phosphate is another important nutrient for plants. It ranged from $0.22 \mathrm{mg} / \mathrm{l}$ in the rainy season to $0.44 \mathrm{mg} / \mathrm{l}$ in the dry season as well as up to $0.5 \mathrm{mg} / \mathrm{l}$ in the study region, with an average of $0.1 \mathrm{mg} / \mathrm{l}$. Both ammonia as well as phosphate levels were higher than the WHO limit but lower than the SON guidelines. As a result, they did not pose a hazard to groundwater quality in terms of the SON criterion (Table 3 and 4). Rocks and minerals decomposition of sewage, agricultural runoff, industrial runoff, aquaculture activities and so on are all sources of phosphate (Olalekan et al., 2018; Olalekan et al., 2021). In groundwater, nitrate and nitrite are two major pollutants, particularly in agricultural areas. Nitrate and nitrite contamination of groundwater is a worldwide issue. High nitrate concentrations in drinking water pose a serious threat to human health, with the main cause being the 
conversion of nitrate to nitrite in the human gut. Drinking water contaminated with nitrates can lead to epidemic disorders like blue baby syndrome in the area (Morufu and Clinton, 2017; Raimi and Sabinus, 2017; Olalekan et al., 2018). The main causes of nitrate contamination are agricultural runoff (cultivation and fertilization), septic tank leaks, landfill leachate as well as municipal rainfall runoff (Isah et al., 2020; Olalekan et al., 2020; Isah et al., 2020; Morufu, 2021; Hussain et al., 2021; Morufu et al., 2021; Hussain et al., 2021). Nitrite $\left(\mathrm{NO}_{3}\right)$, nitrate $\left(\mathrm{NO}_{2}\right)$ and ammonium nitrogen $\left(\mathrm{NH}_{4}{ }^{+}\right)$are all nitrogen derivatives. Fertilizers, organic matters, and several minerals have recently been identified as important nitrite $\left(\mathrm{NO}_{3}\right)$ sources in waters (Olalekan et al., 2020; Raimi et al., 2021). Nitrite $\left(\mathrm{NO}_{3}\right)$ levels of $0.2 \mathrm{mg} / \mathrm{l}$ are considered desirable. The health impact of nitrite causes cyanosis as well as asphyxia (blue-baby syndrome) in infants under three (3) months old. Table 3 and 4 indicate the minimum, maximum, mean, and standard deviation of $\mathrm{NO}_{3}{ }^{-}$concentration in groundwater in the Ebocha-Obrikom area of Rivers State, as determined by laboratory analysis. Under normal conditions, however, $\mathrm{NO}_{3}{ }^{-}$concentrations in groundwater do not surpass $10 \mathrm{mg} / \mathrm{L}$, according to research. In the loess zones, however, $\mathrm{NO}_{3}$ is a frequent pollutant in groundwater. Anthropogenic activities like fertilizer application in agriculture as well as the leaching of sewage wastes or poultry manure are thought to be the cause of high $\mathrm{NO}_{3}$ concentrations in groundwater (Sawyerr et al., 2018; Isah et al., 2020; Olalekan et al., 2020; Isah et al., 2020; Morufu, 2021; Hussain et al., 2021; Morufu et al., 2021; Hussain et al., 2021). The quantities of Nitrite $\left(\mathrm{NO}_{3}\right)$ in the groundwater samples analyzed in this study are high when compared to national standards, with the highest values being 1.79 and $3.30 \mathrm{mg} / \mathrm{L}$ for rainy and dry seasons, respectively. Even so, the mean $\mathrm{NO}_{3}$ concentration is higher than the SON/NAFDAC permitted $\mathrm{NO}_{3}$ level for drinking limit. As a result, daily drinking water intake of high nitrate groundwater poses a risk to local residents' health (Morufu and Clinton, 2017; Raimi and Sabinus, 2017; Olalekan et al., 2018; Raimi et al., 2019; Olalekan et al., 2019; Suleiman et al., 2019; Olalekan et al., 2020). Consequently, increased concentration of $\mathrm{NO}_{3}$ in supplies of groundwater may potentially lead to a health problem toward humankind for instance low levels of oxygen in infants' blood, as well as it is well recognized as methemoglobinemia (Camargo and Alonso, 2006). Nickel generates numerous major adverse health consequences like reduced lung function, chronic bronchitis along with lung cancer as well as nasal sinus. It could also induce reproductive, genotoxicity, neurologic, immunotoxicity, haematotoxicity and developmental effects. The utmost poisonous nickel compound is nickel carbonyl. Hence, the majority of confined groundwater in the research area is therefore fresh as well as soft, implying desirable quality water aimed at human community utilization in terms of the major solute chemistry. While confined groundwater quality by the anthropogenic factors was not affected, the natural hazardous elements together with turbidity, TSS, BOD, DO, COD, Cadmium, Magnesium, Iron, Chromium, Lead as well as Nickel exceeded the required drinking purpose limit as well as would hypothetically threaten the human society. Hence, when water is utilized as a domestic water resource, these potential toxic elements should be paid attention to. So far, not all confined groundwater in the study area pose a health risk to humans, emphasis must remain concentratedly focused on the places where the toxic elements pose a potential threat. In general, the quality of groundwater in this area is quite low as well as not appropriate for drinking. For the quality of groundwater in these locations, the high concentrations of the above-mentioned criteria are accountable. 
Thus, poor quality groundwater will therefore affect the health of indigenous residents who utilize untreated groundwater for cooking, drinking or other household uses will remain impacted. The primary human health impacts of pollutants are shown diagrammatically as follows (see figure 3 below).

Hence, there is need for the oil and gas company to support groundwater exploitation management in the study area and to fully implement centralized supply of water in Ebocha-Obrikom areas of Rivers State, as well as encourage indigenous residents to decrease their usage of untreated groundwater to guarantee residents' safety of drinking water.

\section{Conclusion And Implications}

The comprehensive yearly industrial chemicals production has doubled to almost 2.3 billion tonnes since the turn of the $21^{\text {st }}$ century, and is expected to rise by $85 \%$ come 2030 . As a result, environmental and water contamination are likely to worsen unless there is a swift shift in consumption and production habits, as well as a political commitment to true sustainable water management that completely respects nature. The environmental pressure has increased as a result of the recent global pandemic scenario created by COVID-19, which has intensified waste release. In the Ebocha-Obrikom oil and gas producing area of River State, Nigeria, an integrated approach was used to successfully establish and identify overall suitability assessment of groundwaters for both drinking as well as domestic purposes. Since, groundwater in Ebocha-Obrikom is an important natural resource that is mostly used for domestic as well as agricultural purposes. Overall, a higher percentage of the groundwaters examined are unsuitable for drinking as well as domestic use. Because the quality of water suitability is tied to the water ingested or consumed. So as to produce consistent outcomes aimed at decision making in the face of uncertainty surrounding groundwater threat. the water quality within the research area has been determined to be impaired owing to high levels of physico-chemical and heavy metals parameters (for example Turbidity, DO, BOD, COD, TSS, Magnesium, Iron, Cadmium, Lead, Chromium, Nickel etc.); hence, these poor drinking water quality parameters remain seen as a core influencer. The following highlights are offered after the overall research findings:

- Physicochemical analysis thus revealed that some of physicochemical parameter's remain within recommended standards, notwithstanding most of the water samples having concentrations of Turbidity, DO, BOD, COD, Alkalinity, TSS, Magnesium, Iron, Cadmium, Lead, Chromium, Ammonia, Phosphate, Nitrite and Nickel etc. remain above the recommended standard, describing such waters as unfit for drinking and domestic purposes.

- As a result, under current market economy circumstances, the pollution problem is unlikely to be resolved spontaneously. it is vital that policy instruments should remain used in controlling pollution from this point forward. Thus, it is imperative to identify the environmental policy instruments aimed at improving people's welfare while taking into account the capabilities of relevant stakeholders and putting in place a systematic manner. 
- Human interference (indiscriminate discharge of drainage wastes as well as unregulated use of industrial and agricultural chemicals) must remain more effectively as well as strictly managed, as this is the utmost significant approach for preventing groundwater pollution.

- It is critical to establish a frequent groundwater quality monitoring network in order to understand the causes as well as sources of groundwater contamination on a regular basis so that appropriate remedial actions may be taken in a timely manner.

- Improving groundwater quality monitoring network is advised by creating and raising public awareness (through television, radio, print media and internet). Thus, these strategic measures help decision makers in the development as well as management of groundwater resources in a sustainable manner.

- The obligation for implementing adequate management measures toward improving the quality of groundwater must be assumed on the civic authorities.

- Other Niger Delta States experiencing similar difficulties will benefit from the study's recommendations.

\section{Declarations}

Acknowledgments: I thank Prof. Mynepalli K.C. Sridhar, Prof. Oyeyemi Abisoye S, Prof. Kalada G. Mcfubara, Prof. B.E. Akpan, Prof. I.M. Aprioku as well as all anonymous reviewers, for feedback and discussions that helped to substantially improve this manuscript.

Funding: The water sampling and analysis was supported by AGIP Research Department.

Conflict of Interest: The authors declare that no commercial or financial relationships existed that could be construed as a potential conflict of interest when conducting the research.

Data Availability Statement: The data that support the study findings are summarized in the various tables and are freely available without undue reservation from the corresponding author while the data is submitted to the Figshare web portal:

https://doi.org/10.6084/m9.figshare.14273234.v1.

\section{Supplementary Material}

The Supplementary Material for this article can be found online at: Raimi, Morufu (2021): Water Quality Parameters in Gas Flaring Area of Ebocha-Obrikom of Rivers State, Nigeria. Figshare. Dataset.

https://doi.org/10.6084/m9.figshare.14273234.v1. https://figshare.com/s/c7f5d0e75e096a20a8e1.

\section{References}


1. Adedoyin OO, Olalekan RM, Olawale SH, et al (2020). A review of environmental, social and health impact assessment (Eshia) practice in Nigeria: a panacea for sustainable development and decision making. MOJ Public Health. 2020;9(3):81-87. DOI:

10.15406/mojph.2020.09.00328.https://medcraveonline.com/MOJPH/MOJPH-09-00328.pdf.

2. Adewumi, A. J., \& Laniyan, T. A. (2020). Ecological and human health risks associated with metals in water from Anka Artisanal Gold Mining Area, Nigeria. Human and Ecological Risk Assessment. https://doi.org/10.1080/10807039.2019.1710694.

3. Afolabi Abiodun Segun, Raimi Morufu Olalekan (2021) When Water Turns Deadly: Investigating Source Identification and Quality of Drinking Water in Piwoyi Community of Federal Capital Territory, Abuja Nigeria. Online Journal of Chemistry, 2021, 1, 38-58; DOI: 10.31586/ojc.2021.010105. .

4. Ajayi Folajimi Ajibola, Raimi Morufu Olalekan, Steve-Awogbami Oluseyi Catherine, Adeniji Anthony Olusola, Adebayo Patrick Adekunle (2020) Policy Responses to Addressing the Issues of Environmental Health Impacts of Charcoal Factory in Nigeria: Necessity Today; Essentiality Tomorrow. Communication, Society and Media. Vol 3, No 3. DOI: https://doi.org/10.22158/csm.v3n3p1. http://www.scholink.org/ojs/index.php/csm/article/view/2940.

5. Akerstrom M, Barregard L, Lundh T, Sallsten G. 2013. The relationship between cadmium in kidney and cadmium in urine and blood in an environmentally exposed population. Toxicol Appl Pharmacol 268(3):286-293, PMID: 23454399, https://doi.org/10.1016/j.taap.2013.02.009.

6. Alagoa, E. J and Derefaka, A. A (2002) The Land and People of Rivers State: Eastern Niger Delta. Port Harcourt. Onyoma Research Publications. Pp.8-15.

7. Alagoa, E. J (2005). A History of the Niger Delta: An Historical Interpretation of ljo Oral Tradition. Port Harcourt. Onyoma Research Publications. Pp.. 25-36.

8. American Public Health Association (APHA) (2012) Standard methods for the examination of water and wastewater, 22nd edn. American Public Health Association, Washington, DC.

9. Bernard A. (2008). Cadmium \& its adverse effects on human health. Indian J Med Res 128(4): 55764.

10. Cervantes Gracia K, Llanas-Cornejo D, Husi H. (2017). CVD and oxidative stress. J Clin Med 6(2):22, https://doi.org/10.3390/jcm6020022.

11. Cervantes, C., Campos-García, J., Devars, S., Gutiérrez-Corona, F., Loza Tavera, H., Torres-Guzmán, J. C., \& Moreno-Sánchez, R. (2001). Interactions of chromium with microorganisms and plants. FEMS microbiology reviews, 25(3), 335-347. doi: 10.1111/j.1574-6976.2001.tb00581.x.

12. Chakraborty S, Dutta AR, Sural S, Gupta D, Sen S. (2013). Ailing bones and failing kidneys: a case of chronic cadmium toxicity. Ann Clin Biochem 50(5): 492-495.

13. Chowdhury R, Ramond A, O'Keeffe LM, Shahzad S, Kunutsor SK, Muka T, et al. (2018). Environmental toxic metal contaminants and risk of cardiovascular disease: systematic review and meta-analysis. BMJ 362:k3310, PMID: 30158148, https://doi.org/10.1136/bmj.k3310. 
14. Chukwu, O. (2008). Analysis of Groundwater Pollution from Abattoir waste in Minna. Nigeria. Research Journal of Diary Science, 2(4):74-77.

15. Dami, A., Ayuba H. K. and Amukali, O. (2012). Effects of Gas Flaring and Oil Spillage on Rainwater Collected for Drinking in Okpai and Beneku, Delta State, Nigeria. Global Journal of Human Social Sciences, 12(13): 7-10.

16. Deinkuro Nimisngha Sanchez, Charles W. Knapp, Raimi Morufu Olalekan, Nimlang Henry Nanalok (2021) Oil Spills in the Niger Delta Region, Nigeria: Environmental Fate of Toxic Volatile Organics. 28 June 2021, PREPRINT (Version 1) available at Research Square. DOI: https://doi.org/10.21203/rs.3.rs-654453/v1.

17. Duan N, Wang XL, Liu XD, Lin C, Hou J. (2010). Effect of anaerobic fermentation residues on a chromium-contaminated soil-vegetable system. Procedia Environmental Sciences 2: 1585-1597.

18. Egbueri, J. C., \& Unigwe, C. O. (2020). Understanding the extent of heavy metal pollution in drinking water supplies from Umunya, Nigeria: An indexical and statistical assessment. Analytical Letters, 53(13), 2122-2144. https://doi.org/10.1080/00032719.2020. 1731521.

19. Ezenwaji, E. E., \& Ezenweani, I. D. (2018). Spatial analysis of groundwater quality in Warri Urban Nigeria. Sustainable Water Resources and Management, 1, 2. https://doi.org/10.1007/ s40899-0180264-2.

20. Food and Agriculture Organization of the United Nations (FAO) and United Nations Environment Programme (UNEP) (2021) Global assessment of soil pollution". Summary for Policy Makers. Rome.

21. Ghani A. (2011). Effect of chromium toxicity on growth, chlorophyll and some mineral nutrients of Brassica juncea L. Egyptian Acad J Biol Sci 2(1): 9-15.

22. Gift RA, Olalekan RM, Owobi OE, Oluwakemi RM, Anu B, Funmilayo AA (2020). Nigerians crying for availability of electricity and water: a key driver to life coping measures for deepening stay at home inclusion to slow covid-19 spread. Open Access Journal of Science. 2020;4(3):69-80. DOI: 10.15406/oajs.2020.04.00155.

23. Gift R A, Olalekan RM (2020). Access to electricity and water in Nigeria: a panacea to slow the spread of Covid-19. Open Access J Sci. 2020;4(2):34. DOI: 10.15406/oajs.2020.04.00148. https://medcrave.com/index.php?/articles/det/21409/.

24. Goyer RA. (1990). Lead toxicity: from overt to subclinical to subtle health effects. Environ Health Perspect. 86: 177-181.

25. Hasan, M.M. \& Gerber, N. (2016). The impacts of piped water on water quality, sanitation, hygiene and health in rural households of north-western Bangladesh -a quasi-experimental analysis. ZEF Discussion Papers 217.

26. Henry Olawale Sawyerr, Morufu Olalekan Raimi, Adedotun Timothy Adeolu \& Oluwaseun Emmanuel Odipe (2019) Measures of Harm from Heavy Metal Pollution in Battery Technicians' Workshop within Ilorin Metropolis, Kwara State, Nigeria. Scholink Communication, Society and Media ISSN 2576-5388 (Print) ISSN 2576-5396 (Online) Vol. 2, No. 2, 2019 www.scholink.org/ojs/index.php/csm. DOI: https://doi.org/10.22158/csm.v2n2p73. 
27. Henry O. Sawyerr, Odipe E. Oluwaseun1, Olawale S. Asabi, Raimi M. Olalekan (2019) Bacteriological Assessment of Selected Hand Dug Wells in Students' Residential Area: A Case Study of Osun State College of Health Technology, Ilesa, Nigeria. Global Scientific Journal, Volume 7, Issue 1, January 2019, Online: ISSN 2320-9186. .

28. Hu XF, Singh K, Chan HM. (2018). Mercury exposure, blood pressure, and hypertension: a systematic review and dose-response meta-analysis. Environ Health Perspect 126(7):076002, PMID: 30073953, https://doi.org/10.1289/EHP2863.

29. Hussain Muhammad Isah, Morufu Olalekan Raimi, Henry Olawale Sawyerr (2021) Patterns of Chemical Pesticide Use and Determinants of Self-Reported Symptoms on Farmers Health: A Case Study in Kano State for Kura Local Government Area of Nigeria. Research on World Agricultural Economy. Vol 2, No. 1. DOI: http://dx.doi.org/10.36956/rwae.v2i1.342. http://ojs.nassg.org/index.php/rwae/issue/view/31

30. Hussain Muhammad Isah, Morufu Olalekan Raimi, Henry Olawale Sawyerr (2021) Probabilistic Assessment of Self-Reported Symptoms on Farmers Health: A Case Study in Kano State for Kura Local Government Area of Nigeria. Research on World Agricultural Economy. Vol 2, No. 1. DOI: http://dx.doi.org/10.36956/rwae.v2i1.336. http://ojs.nassg.org/index.php/rwaecn/article/view/336/pdf.

31. Isah, H. M., Sawyerr, H. O., Raimi, M. O., Bashir, B. G., Haladu, S. \& Odipe, O. E. (2020). Assessment of Commonly Used Pesticides and Frequency of Self-Reported Symptoms on Farmers Health in Kura, Kano State, Nigeria. Journal of Education and Learning Management (JELM), HolyKnight, vol. 1, 3154. doi.org/10.46410/jelm.2020.1.1.05. https://holyknight.co.uk/journals/jelm-articles/.

32. Isah Hussain Muhammad, Raimi Morufu Olalekan, Sawyerr Henry Olawale, Odipe Oluwaseun Emmanuel, Bashir Bala Getso, Suleiman Haladu (2020) Qualitative Adverse Health Experience Associated with Pesticides Usage among Farmers from Kura, Kano State, Nigeria. Merit Research Journal of Medicine and Medical Sciences (ISSN: 2354-323X) Vol. 8(8) pp. 432-447, August, 2020. DOI: 10.5281/zenodo.4008682.

https://meritresearchjournals.org/mms/content/2020/August/lsah\%20et\%20al.htm.

33. Islam, S., Bhuiyan, M., Rume, T., \& Azam, G. (2017). Hydrogeochemical investigation of groundwater in shallow coastal aquifer of Khulna District, Bangladesh. Applied Water Science, 7, 1-18.

34. Koleayo Oluwafemi Omoyajowo, Morufu Olalekan Raimi, Temitope Oluwagbenga Waleola, Oluwaseun Emmanuel Odipe, Amos Lanrewaju Ogunyebi (2021) Public Health Knowledge and Perception of Microplastics Pollution: Lessons from the Lagos Lagoon, 10 May 2021, PREPRINT (Version 1) available at Research Square [https://doi.org/10.21203/rs.3.rs-506361/v1].

35. Lanphear BP, Rauch S, Auinger P, Allen RW, Hornung RW. (2018). Low-level lead exposure and mortality in US adults: a population-based cohort study. Lancet Public Health 3(4):e177-e184, PMID: 29544878, https://doi.org/10.1016/S2468- 2667(18)30025-2.

36. Liu T, Zhang M, Guallar E, Wang G, Hong X, Wang X, et al. (2019). Trace minerals, heavy metals, and preeclampsia: findings from the Boston Birth Cohort. J Am Heart Assoc 8(16):e012436, PMID: 
31426704, https://doi.org/10.1161/JAHA.119. 012436.

37. Martin S, Griswold W. (2009). Human health effects of heavy metals. Environmental Science and Technology Briefs for Citizens (15): 1-6.

38. Malcolm, I. A., Youngson, A. F., and Soulsby, C. (2003). Survival of salmonid eggs in a degraded gravel-bed stream: effects of groundwater - surface water interactions. River Res. Appl. 19, 303-316. doi: $10.1002 /$ rra.706.

39. Mohanty M, Kumar Patra H. (2013). Effect of ionic and chelate assisted hexavalent chromium on mung bean seedlings (Vigna Radiata I. Wilczek. Var k-851) during seedling growth. JSPB 9(2): 232241.

40. Morrison, G., O., Fatoki, O., S and Ekberg, A., 2001. Assessment of the Impact of Point Source Pollution from the Keiskammahoek Sewage Treatment Plant on the Keiskamma River. Water South Africa, 27: 475-480.

41. Morufu Olalekan Raimi, Aziba-anyam Gift Raimi and Teddy Charles Adias (2021) 'Silent Pandemic': Evidence-Based Environmental and Public Health Practices to Respond to the Covid-19 Crisis. IntechOpen. DOI: http://dx.doi.org/10.5772/intechopen.100204. ISBN 978-1-83969-144-7. https://www.intechopen.com/online-first/silent-pandemic-evidence-based-environmental-and-publichealth-practices-to-respond-to-the-covid-19-

42. Morufu Olalekan Raimi, Aziba-anyam Gift Raimi, Teddy Charles Adias (2021). Evidence-based Environmental and Public Health Practices to Respond to the COVID-19 Crisis, 07 May 2021, PREPRINT (Version 1) available at Research Square [https://doi.org/10.21203/rs.3.rs-504983/v1] https://europepmc.org/article/PPRID/PPR335534; EMSID:EMS123969.

43. Morufu Olalekan Raimi, Tonye Vivien Odubo, Ogah Alima, Henry Akpojubaro Efegbere, Abinotami Williams Ebuete (2021) Articulating the effect of Pesticides Use and Sustainable Development Goals (SDGs): The Science of Improving Lives through Decision Impacts. Research on World Agricultural Economy. Vol 2, No. 1. DOI: http://dx.doi.org/10.36956/rwae.v2i1.347. http://ojs.nassg.org/index.php/rwae/issue/view/31.

44. Morufu Olalekan Raimi, Ebikapaye Okoyen, Tuebi Moses, Aziba-anyam Gift Raimi, Adedoyin Oluwatoyin Omidiji, Aishat Funmilayo Abdulraheem, Mariam Oluwakemi Raimi, Beatrice Oka Joseph (2021) Do Weak Institutions Prolong Crises? [\#ENDSARs] in the Light of the Challenges and opportunities beyond COVID-19 Pandemic and the Next Normal in Nigeria. Communication, Society and Media. ISSN 2576-5388 (Print) ISSN 2576-5396 (Online) Vol. 4, No. 2, DOI: https://doi.org/10.22158/csm.v4n2p1. http://www.scholink.org/ojs/index.php/csm/article/view/3790.

45. Morufu Olalekan Raimi, Tonye Vivien Odubo \& Adedoyin Oluwatoyin Omidiji (2021) Creating the Healthiest Nation: Climate Change and Environmental Health Impacts in Nigeria: A Narrative Review. Scholink Sustainability in Environment. ISSN 2470-637X (Print) ISSN 2470-6388 (Online) Vol. 6, No. 1, 2021 www.scholink.org/ojs/index.php/se. URL: http://dx.doi.org/10.22158/se.v6n1p61. http://www.scholink.org/ojs/index.php/se/article/view/3684. 
46. Morufu Olalekan Raimi (2021). "Self-reported Symptoms on Farmers Health and Commonly Used Pesticides Related to Exposure in Kura, Kano State, Nigeria". Annals of Community Medicine \& Public Health. 1(1): 1002. http://www.remedypublications.com/open-access/self-reported-symptoms-onfarmers-health-and-commonly-used-pesticides-related-6595.pdf.

http://www.remedypublications.com/annals-of-community-medicine-public-health-home.php.

47. Morufu Raimi and Clinton Ezekwe (2017), Assessment of Trace Elements in Surface and Ground Water Quality (2017) LAP Lambert Academic Publishing. Mauritius. ISBN: 978-3-659-38813-2. .

48. Mostafa, M.G., Helal Uddin, S.M., Haque, A.B.M.H., (2017). Assessment of hydrogeochemistry and groundwater quality of Rajshahi City in Bangladesh. Appl Water Sci 7, 4663-4671. https://doi.org/10.1007/s13201-017-0629-y.

49. Mudgal V, Madaan N, Mudgal A, Singh RB, Mishra S. (2010). Effect of toxic metals on human health. Open Nutraceuticals J 3: 94-99.

50. Nath K, Shyam S, Singh D, Shanna YK. (2008). Effect of chromium and tannery effluent toxicity on metabolism and growth in cowpea (Vigna sinensis L. Saviex Hassk) seedling. Res Environ Life Sci 1: 91-94.

51. Nordberg GF, Nogawa K, Nordberg M. (2015). Cadmium. In: Handbook on the Toxicology of Metals. Nordberg GF, Nordberg M, eds. Amsterdam, Netherlands: Elsevier, 667-716.

52. Odipe O. E, Raimi M. O, Suleiman F (2018). Assessment of Heavy Metals in Effluent Water Discharges from Textile Industry and River Water at Close Proximity: A Comparison of Two Textile Industries from Funtua and Zaria, North Western Nigeria. Madridge Journal of Agriculture and Environmental Sciences. 2018; 1(1): 1-6.doi: 10.18689/mjaes-1000101. https://madridge.org/journal-of-agricultureand-environmental-sciences/mjaes-1000101.php.

53. Odubo Tonbra Robert and Raimi Morufu Olalekan (2019) Resettlement and Readjustment Patterns of Rural Dwellers During and After Flood Disasters in Bayelsa State Nigeria. British Journal of Environmental Sciences Vol.7, No .3, Pp. 45-52, July 2019. .

54. Ogoni, H. A (2010) Ethical Perspective of Oil and Gas Exploration in Nigeria in Nigeria 5th Anniversary of Hoscom of Nigeria Oil and Gas.

55. Okoyen E, Raimi M O, Omidiji A O, Ebuete A W (2020). Governing the Environmental Impact of Dredging: Consequences for Marine Biodiversity in the Niger Delta Region of Nigeria. Insights Mining Science and technology 2020; 2(3): 555586. DOI: 10.19080/IMST.2020.02.555586.

56. Olalekan Morufu Raimi, Abiola llesanmi, Ogah Alima, Dodeye E. Omini (2021) Exploring How Human Activities Disturb the Balance of Biogeochemical Cycles: Evidence from the Carbon, Nitrogen and Hydrologic Cycles. Research on World Agricultural Economy. Volume 02, Issue 03. DOI: http://dx.doi.org/10.36956/rwae.v2i3.426. http://ojs.nassg.org/index.php/rwae.

57. Olalekan RM, Oluwatoyin OA, Olawale SH, Emmanuel OO, Olalekan AZ (2020) A Critical Review of Health Impact Assessment: Towards Strengthening the Knowledge of Decision Makers Understand Sustainable Development Goals in the Twenty-First Century: Necessity Today; Essentiality Tomorrow. 
Research and Advances: Environmental Sciences. 2020(1): 72-84. DOI: 10.33513/RAES/2001-13. https://ospopac.com/journal/environmental-sciences/early-online.

58. Olalekan R. M, Oluwatoyin O and Olalekan A (2020) Health Impact Assessment: A tool to Advance the Knowledge of Policy Makers Understand Sustainable Development Goals: A Review. ES Journal of Public Health; 1(1); 1002. https://escientificlibrary.com/public-health/in-press.php.

59. Olalekan R. M, Dodeye E. O, Efegbere H. A, Odipe O. E. Deinkuro N. S, Babatunde A and Ochayi E. O (2020) Leaving No One Behind? Drinking-Water Challenge on the Rise in Niger Delta Region of Nigeria: A Review. Merit Research Journal of Environmental Science and Toxicology (ISSN: 23502266) Vol. 6(1): 031-049 DOl: 10.5281/zenodo.3779288.

60. Olalekan RM, Muhammad IH, Okoronkwo UL, Akopjubaro EH (2020). Assessment of safety practices and farmer's behaviors adopted when handling pesticides in rural Kano state, Nigeria. Arts \& Humanities Open Access Journal. 2020;4(5):191-201. DOI: 10.15406/ahoaj.2020.04.00170.

61. Olalekan RM, Adedoyin OO, Ayibatonbira A, et al (2019). "Digging deeper" evidence on water crisis and its solution in Nigeria for Bayelsa state: a study of current scenario. International Journal of Hydrology. 2019;3(4):244-257. DOI: 10.15406/ijh.2019.03.00187.

62. Olalekan R. M, Vivien O. T, Adedoyin O. O, et al. (2018). The sources of water supply, sanitation facilities and hygiene practices in oil producing communities in central senatorial district of Bayelsa state, Nigeria. MOJ Public Health. 2018;7(6):337-345. DOI: 10.15406/mojph.2018.07.00265.

63. Olalekan, R. M., Omidiji, A. O., Nimisngha, D., Odipe, O. E. and Olalekan, A. S. (2018). Health Risk Assessment on Heavy Metals Ingestion through Groundwater Drinking Pathway for Residents in an Oil and Gas Producing Area of Rivers State, Nigeria. Open Journal of Yangtze Gas and Oil, 3, 191206. https://doi.org/10.4236/ojogas.2018.33017.

64. Oluwaseun Emmanuel Odipe., Raimi Morufu Olalekan, Nimisingha Deinkuro Sanchez, Abdulraheem Aishat Funmilayo, Okolosi-Patainnocent Edewor, Habeeb Modupe Lateefat and Mary Fadeyibi (2019) Assessment of Environmental Sanitation, Food Safety Knowledge, Handling Practice among Food Handlers of Bukateria Complexes in lju Town, Akure North of Ondo-State, Nigeria. Acta Scientific Nutritional Health 3.6 (2019): 186-200. DOI: 10.31080/ASNH.2019.03.0308.

65. Omidiji A. $O$ and Raimi M. O (2019) Practitioners Perspective of Environmental, Social and Health Impact Assessment (ESHIA) Practice in Nigeria: A Vital Instrument for Sustainable Development. Paper Presented at the Association for Environmental Impact Assessment of Nigeria (AEIAN) On Impact Assessment: A Tool for Achieving the Sustainable Development Goals in Nigeria, 7th and 8th November, 2019 In University of Port Harcourt. https://aeian.org/wp-content/uploads/2019/08/EIAPresentations-Portharcourt.pdf.

66. Omotoso Ayodele Jacob, Omotoso Elizabeth Anuoluwa, Morufu Olalekan Raimi (2021) Potential Toxic levels of Cyanide and Heavy Metals in Cassava Flour Sold in Selected Markets in Oke Ogun Community, Oyo State, Nigeria, 01 July 2021, PREPRINT (Version 1) available at Research Square [https://doi.org/10.21203/rs.3.rs-658748/v1]. 
67. Onyemesili, O. O., Egbueri, J. C., \& Ezugwu, C. K. (2020). Assessing the pollution status, ecological and health risks of surface waters in Nnewi urban, Nigeria: Implications of poor waste disposal. Environmental Forensics. https://doi.org/10.1080/15275922.2020. 1850564.

68. Phippen B, Horvath C, Nordin R, Nagpal N. (2008). Ambient water quality guidelines for iron: overview. Ministry of Environment Province of British Columbia.

69. Premoboere Edna Ateboh and Raimi Morufu Olalekan (2018). Corporate Civil Liability and Compensation Regime for Environmental Pollution in the Niger Delta. International Journal of Recent Advances in Multidisciplinary Research. Vol. 05, Issue 06, pp. 3870-3893, June, 2018.

70. Raimi, M. O., Mcfubara, K. G., Abisoye, O. S., Ifeanyichukwu Ezekwe, C., Henry Sawyerr, O., \& Raimi, G. A-anyam (2021) Responding to the call through Translating Science into Impact: Building an Evidence-Based Approaches to Effectively Curb Public Health Emergencies [COVID-19 Crisis]. Global Journal of Epidemiology and Infectious Disease, 1(1). DOI: 10.31586/gjeid.2021.010102. Retrieved from https://www.scipublications.com/journal/index.php/gjeid/article/view/72.

71. Raimi Morufu Olalekan, Emeka Chisom Lucky, Ebikapaye Okoyen, Angalabiri Clement, Christopher Ogbointuwe, Atoyebi Babatunde (2021) COVID-19 Decision Impacts: Vaccine Hesitancy, its Barriers and Impact Studies: Taking Bayelsa State as an Example., 27 May 2021, PREPRINT (Version 1) available at Research Square [https://doi.org/10.21203/rs.3.rs-566532/v1]

72. Raimi Morufu Olalekan, Ayinla Lateefat Olajumoke, Ogah Alima (2021) First to Respond, Last to Leave: The Role of Para-Military Agencies in Disaster Management: Evidence from Nigeria. Sumerianz Journal of Medical and Healthcare, 2021, Vol. 4, No. 2, pp. 96-100 ISSN(e): 2663-421X, ISSN(p): 2706-8404 Website: https://www.sumerianz.com DOI:

https://doi.org/10.47752/sjmh.42.96.100. https://www.sumerianz.com/?ic=journalhome\&journal=31\&info=archive-detail\&month=06-2021\&issue=2\&volume=4.

73. Raimi Morufu Olalekan, Abiola Ilesanmi, Ogah Alima, Dodeye E. Omini and Aziba-anyam Gift Raimi (2021) Exploring How Human Activities Disturb the Balance of Biogeochemical Cycles: Evidence from the Carbon, Nitrogen and Hydrologic Cycles. IntechOpen. DOI: http://dx.doi.org/10.5772/intechopen.98533. https://www.intechopen.com/online-first/77696.

74. Raimi Morufu Olalekan \& Raimi Aziba-anyam Gift (2020). The Toughest Triage in Decision Impacts: Rethinking Scientific Evidence for Environmental and Human Health Action in the Times of Concomitant Global Crises. CPQ Medicine, 11(1), 01-05.

75. Raimi Morufu Olalekan, Moses Tuebi, Okoyen Ebikapaye, Sawyerr Henry Olawale, Joseph Beatrice Oka, Oyinlola Bilewu Olaolu (2020) “A Beacon for Dark Times: Rethinking Scientific Evidence for Environmental and Public Health Action in the Coronavirus Diseases 2019 Era" Medical and Research Microbiology, Vol. 1, Issues 3.

76. Raimi Morufu Olalekan, Ihuoma Blossom Adindu, Esther Onyinyechi Udensin, Abdulraheem Aishat Funmilayo, Opufou Tarekebi, Deinkuro Nimisingha Sanchez, Adebayo Patrick Adekunle and Adeniji Anthony Olusola (2020) "Health Impact Assessment: Expanding Public Policy Tools for Promoting 
Sustainable Development Goals (SDGs) in Nigeria". EC Emergency Medicine and Critical Care 4.9 (2020).

77. Raimi Morufu Olalekan, Sawyerr Henry Olawale and Isah Hussain Muhammad (2020) Health risk exposure to cypermethrin: A case study of kano state, Nigeria. Journal of Agriculture. 7th International Conference on Public Healthcare and Epidemiology. September 14-15, 2020 | Tokyo, Japan.

78. Raimi Morufu Olalekan (2019) 21st Century Emerging Issues in Pollution Control. 6th Global Summit and Expo on Pollution Control May 06-07, 2019 Amsterdam, Netherlands.

79. Raimi Morufu Olalekan, Omidiji Adedoyin O, Adeolu Timothy Adedotun, Odipe Oluwaseun Emmanuel and Babatunde Anu (2019) An Analysis of Bayelsa State Water Challenges on the Rise and Its Possible Solutions. Acta Scientific Agriculture 3.8 (2019): 110-125. DOI: 10.31080/ASAG.2019.03.0572.

80. Raimi M O, Suleiman R M, Odipe O E, Salami J T, Oshatunberu M, et al (2019). Women Role in Environmental Conservation and Development in Nigeria. Ecology \& Conservation Science, 1(2): DOI: 10.19080/ECOA.2019.01.555558. Volume 1 Issue 2 - July 2019. https://juniperpublishers.com/ecoa/pdf/ECOA.MS.ID.555558.pdf.

81. Raimi Morufu Olalekan, Bilewu Olaolu Oyinlola, Adio Zulkarnaini Olalekan, Abdulrahman Halimat (2019) Women Contributions to Sustainable Environments in Nigeria. Journal of Scientific Research in Allied Sciences. 5(4), 35-51. ISSN NO. 2455-5800. DOI No. 10.26838/JUSRES.2019.5.4.104.

82. Raimi M. O, Abdulraheem A. F, Major Iteimowei, Odipe O. E, Isa H. M, Onyeche Chinwendu (2019). The Sources of Water Supply, Sanitation Facilities and Hygiene Practices in an Island Community: Amassoma, Bayelsa State, Nigeria. Public Health Open Access 2019, 3(1): 000134. ISSN: 2578-5001. DOI: $10.23880 /$ phoa-16000134.

83. Raimi Morufu Olalekan, Omidiji Adedoyin O, Abdulraheem Aishat Funmilayo, Ochayi Ekoja Owobi (2018) A Survey of Hand Washing Behaviour and Awareness among Health Care Workers in Health Care Facilities in Kubwa District of Bwari Area Council, F.C.T. Abuja, Nigeria. Annals of Ecology and Environmental Science. Volume 2, Issue 2, 2018, PP 1-18.

84. Raimi Morufu Olalekan, Tonye V. Odubo, Omidiji Adedoyin O, Oluwaseun E. Odipe (2018) Environmental Health and Climate Change in Nigeria. World Congress on Global Warming. Valencia, Spain. December 06-07, 2018.

85. Raimi Morufu Olalekan, and Sabinus Chibuzor Ezugwu. (2017) Influence of Organic Amendment on Microbial Activities and Growth of Pepper Cultured on Crude Oil Contaminated Niger Delta Soil. International Journal of Economy, Energy and Environment. Vol. 2, No. 4, 2017, pp. 56-76. DOI: 10.11648/j.ijeee.20170204.12.

86. Raimi Morufu Olalekan and Sabinus C. E. (2017) An Assessment of Trace Elements in Surface and Ground Water Quality in the Ebocha-Obrikom Oil and Gas Producing Area of Rivers State, Nigeria. International Journal for Scientific and Engineering Research (ljser): Volume 8, Issue 6, July Edition. ISSN: 2229-5518. 
87. Raimi, M. O, Pigha, Tarilayun K and Ochayi, E. O (2017) Water-Related Problems and Health Conditions in the Oil Producing Communities in Central Senatorial District of Bayelsa State. Imperial Journal of Interdisciplinary Research (IJIR). Vol-3, Issue-6, ISSN: 2454-1362.

88. Rodríguez MC, Barsanti L, Passarelli V, Evangelista V, Conforti V, Gualtieri P. (2007). Effects of chromium on photosynthetic and photoreceptive apparatus of the alga Chlamydomonas reinhardtii. Environ Res 105(2): 234-239.

89. Sawyerr O. H, Odipe O. E, Olalekan R. M, et al. (2018) Assessment of cyanide and some heavy metals concentration in consumable cassava flour "lafun" across Osogbo metropolis, Nigeria. MOJ Eco Environ Sci. 2018;3(6):369-372. DOI: 10.15406/mojes.2018.03.00115.

90. Suleiman Romoke Monsurat, Raimi Morufu Olalekan and Sawyerr Henry Olawale (2019) A Deep Dive into the Review of National Environmental Standards and Regulations Enforcement Agency (NESREA) Act. International Research Journal of Applied Sciences. pISSN: 2663-5577, eISSN: 26635585. DOI No. Irjas.2019.123.123. . https://scirange.com/abstract/irjas.2019.108.125.

91. Tellez-Plaza M, Jones MR, Dominguez-Lucas A, Guallar E, Navas-Acien A. 2013a. Cadmium exposure and clinical cardiovascular disease: a systematic review. Curr Atheroscler Rep 15(10):356, PMID: 23955722, https://doi.org/10.1007/s11883-013-0356-2.

92. Tinkov AA, Filippini T, Ajsuvakova OP, Skalnaya MG, Aaseth J, Bjørklund G, et al. 2018. Cadmium and atherosclerosis: a review of toxicological mechanisms and a Meta-analysis of epidemiologic studies. Environ Res 162:240-260, PMID: 29358116, https://doi.org/10.1016/j.envres.2018.01.008.

93. Ukah, B. U., Egbueri, J. C., Unigwe, C. O., \& Ubido, O. E. (2019). Extent of heavy metals pollution and health risk assessment of groundwater in a densely populated industrial area, Lagos, Nigeria. International Journal of Energy and Water Resources, 3(4), 291-303. https://doi.org/10.1007/s42108-019-00039-3

94. USEPA. (2019). United States-Environmental Protection Agency (2019). Regional Screening Levels (RSLs) - Equations https://www.epa.gov/risk/regional-screening levels-rsls-equations (accessed 26.08.19).

95. USEPA (US Environmental Protection Agency) (2017) National recommended water quality criteriaaquatic life criteria table and human health criteria table. https://www.epa.gov.wqc/nationalrecommended-water-quality criteria-aquatic-life-criteria-table.

96. Valko MMHCM, Morris H, Cronin MTD. (2005). Metals, toxicity and oxidative stress. Curr Med Chem 12(10): 1161-1208.

97. Vuori K-M. (1995). Direct and Indirect effects of iron on river eco systems. Annal Zoo Fennici 32: 317-3.

98. Wolińska A, Stępniewska Z, Włosek R. (2013). The influence of old leather tannery district on chromium contamination of soils, water and plants. Nat Sci 5(2A): 253-258.

99. Yongsheng W, Qihui L, Qian T. (2011). Effect of Pb on growth, accumulation and quality component of tea plant. Procedia Engineering 18: 214-219. 
100. Zayed AM, Terry N. (2003). Chromium in the environment: factors affecting biological remediation. Plant Soil 249(1): 139-156.

101. Zhang W, Ma L, Abuduwaili J, Ge Y, Issanova G, Saparov G (2019) Hydro-chemical characteristics and irrigation suitability of surface water in the Syr Darya River, Kazakhstan. Environ Monit Assess 191:572. https://doi.org/10.1007/s10661-019-7713-8

102. Zhou Y, Li P, Xue L, Dong Z, Li D <bi $>$ et $</$ bi $><b i>a l .</ b i>(2020)$ Solute geochemistry and groundwater quality for drinking and irrigation purposes: a case study in Xinle City, North China. Geochemistry. https://doi.org/ 10.1016/j.chemer.2020.125609.

\section{Figures}

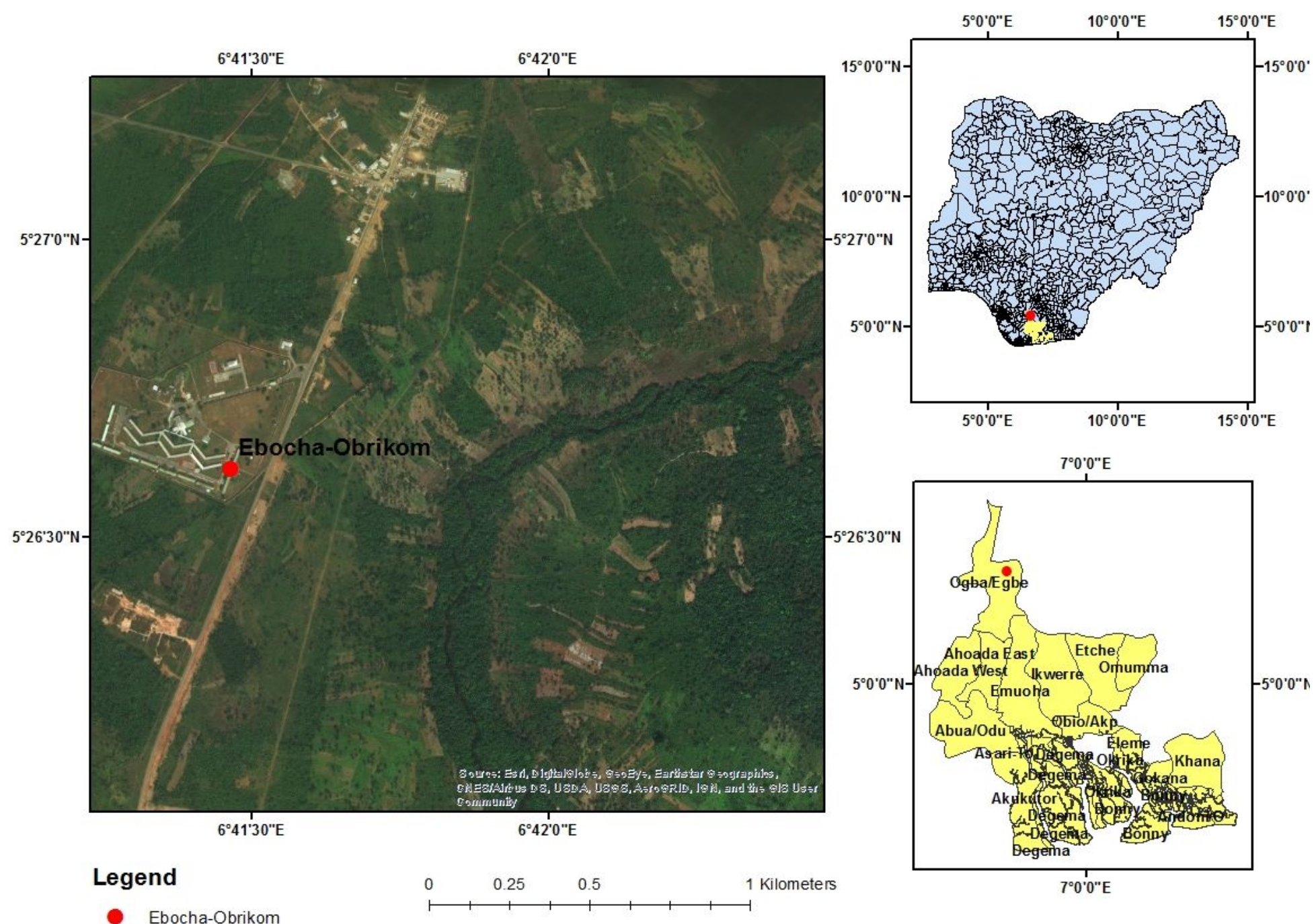

\section{Figure 1}

Map Showing the Study Area with Nigeria and River State insert. Sources: Adapted from Olalekan et al., 2018 [https://doi.org/10.4236/ojogas.2018.33017] 


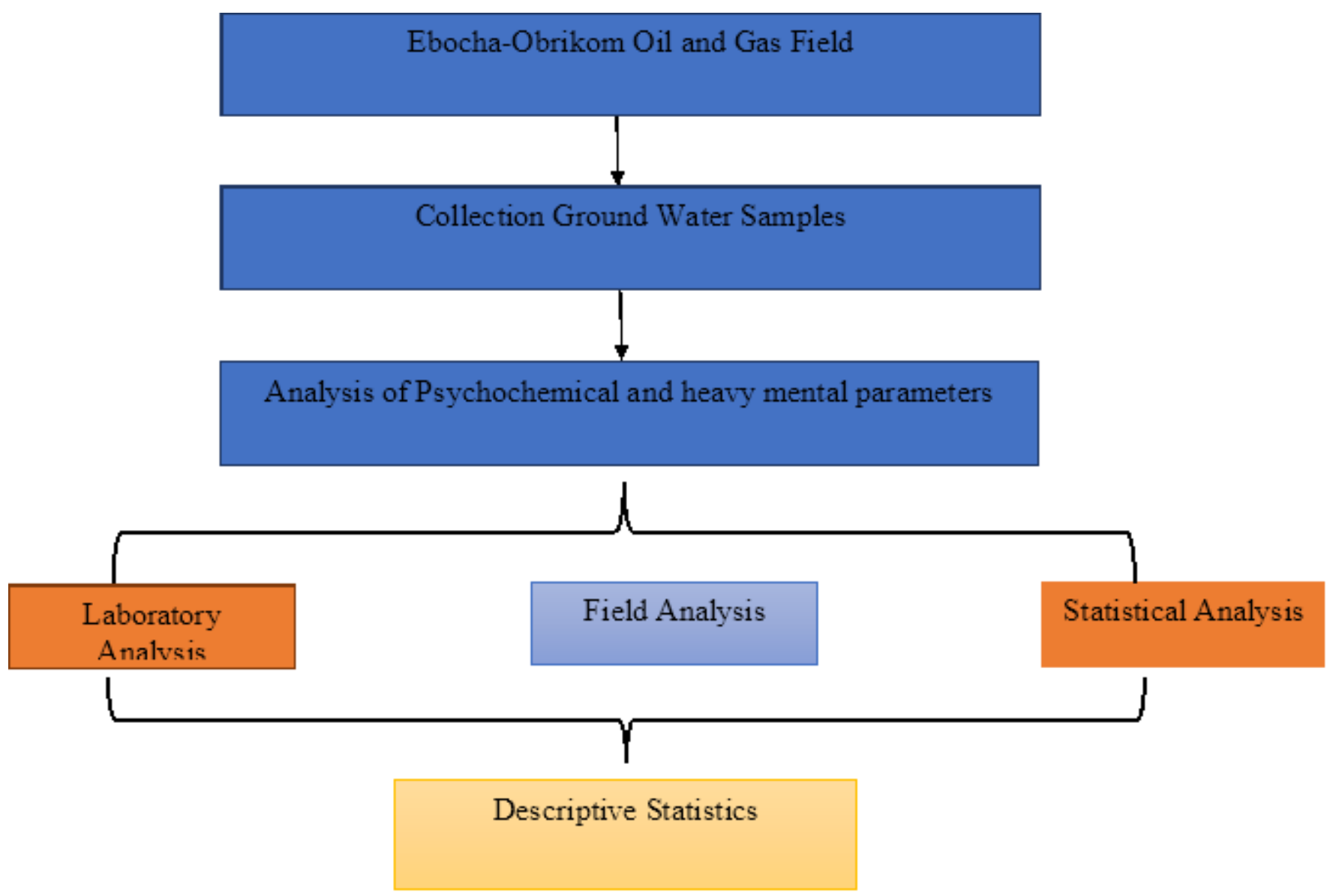

Figure 2

A schematic illustration of quantification methodology adopted for the current study. 


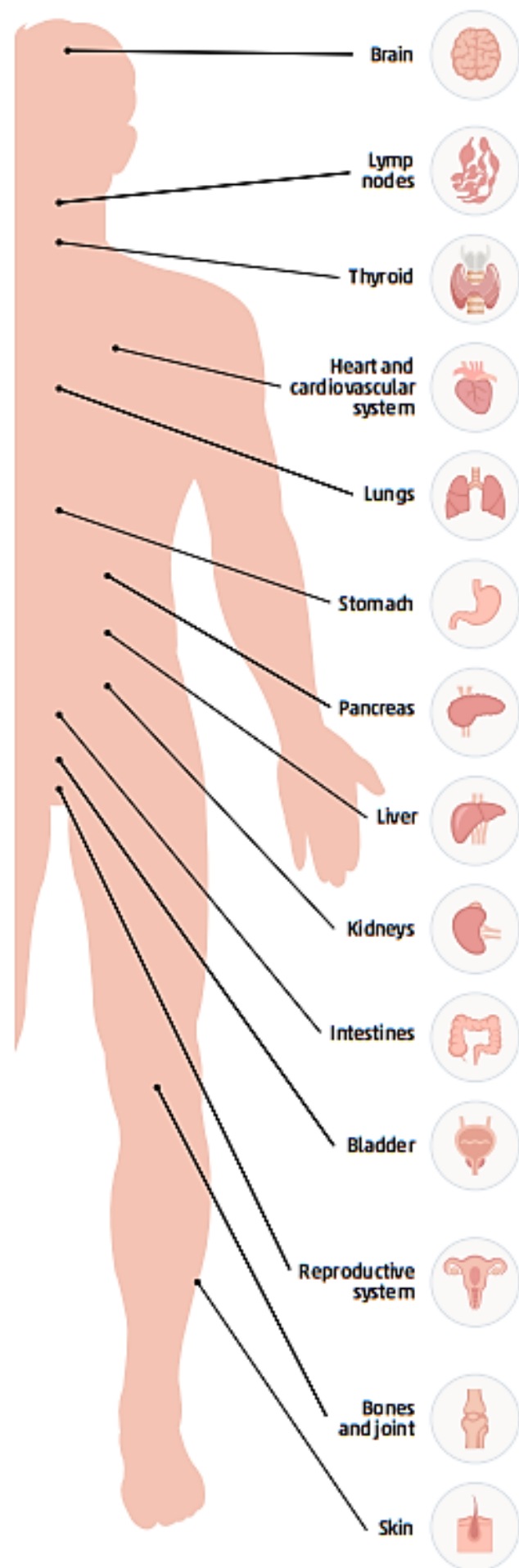

Lead, manganese, mercury, tin, PBDEs, PAHs, PCBs

Neu rodevelopmental impairment, redudion of

intell igence quotient, behavioural disorder,

Parkinson-typesyndrome, headache

BTEX, lead, PFAS, PCBS

Alteredimmune response, reduced response

tovacrines in children

Cadmium, PCBs, PBDEs

Altered metabolismandreproductive hor mone

levels, reduced thyroid hormones, altered growth

Benzene, lead, merary, organochlorine

pestiddes, PAHs, PFAS, PCBs, microplastics

Hypertension, haemolysis, anaemia, cardiovascular

disease, elevated leucocytecount, leukaemia

Arsenic, asbestos, cadmium, chromium,

copper, mercury, radon

Pulmonary emphysema, asthma, chemical pneumonia

lung cancer, mesothelioma

Nitrogen and ioniz ing radiation

Stomachcancer

Phthalates, PCBs

Alteredinsulin metabolismand adipogenesis, diabetes

DDT, chromium, copper, merary, PAHs, PCBS, PFAS,

phthalates

increased cholesterol levels, livercancer, elevated hepatic enzyme levels, necrosis

Cadmium, lead, mercury, PAHs, PFAS

Renal tubular dysfundion, kidney weight changes,

progressive nephropathy, chronic inflammation, kidney

ancer

Arsenic, copper, lead, mercury, tin, microplastics, POPs

Nausea, vomiting, diarrhea, cancer of gastrointestinal

system, abdominal pain and cramp ing

Arsenic, lead

Cancer of urinary bladder, urinary changes

Ant imomy, asbestos, lead, manganese, phthalates,

PBDES, PCBS, PFAS

Testioular atrophy, early menopause, reduced testosterone,

reproductwe alterations, decreased libido, impotence,

sexual dysfunction, endometriosis, hormonal ancers

(breast, prostate, testes), infertility, ovary cancer

Cadmium, lead, PCBs, radium and its decay products

Impaired bones development, slow growth, changes in

metabolism of caldum andbone formation, osteomalasia, bone cancer

Arsenic, chromium, PAHs, PCBs

Hy perkeratosis, typerpigmentation, hypopigmentation, skin irritation and inflammation, chloracne, hirsutism, skin, tooth, and nail abnofma lities

\section{Figure 3}

Main effects of contaminants on human health, indicating the organs or systems affected and the contaminants causing them. Source: Adapted from FAO and UNEP (2021) 